\title{
EXISTENCE OF SOLUTIONS AND SEPARATION FROM SINGULARITIES FOR A CLASS OF FOURTH ORDER DEGENERATE PARABOLIC EQUATIONS
}

\author{
GIULIO SCHIMPERNA AND SERGEY ZELIK
}

\begin{abstract}
A nonlinear parabolic equation of the fourth order is analyzed. The equation is characterized by a mobility coefficient that degenerates at 0 . Existence of at least one weak solution is proved by using a regularization procedure and deducing suitable a priori estimates. If a viscosity term is added and additional conditions on the nonlinear terms are assumed, then it is proved that any weak solution becomes instantaneously strictly positive. This in particular implies uniqueness for strictly positive times and further time-regularization properties. The long-time behavior of the problem is also investigated and the existence of trajectory attractors and, under more restrictive conditions, of strong global attractors is shown.
\end{abstract}

\section{INTRODUCTION}

The present paper is devoted to the analysis of the following class of fourth order parabolic equations:

$$
\begin{aligned}
& u_{t}-\operatorname{div}(b(u) \nabla w)=0, \\
& w=\delta u_{t}-\Delta u+f(u)+\gamma(u)-g,
\end{aligned}
$$

on $\Omega \times(0,+\infty), \Omega$ being a bounded smooth subset of $\mathbb{R}^{d}, d \in\{2,3\}$, coupled with the initial and boundary conditions

$$
\begin{aligned}
& \left.u\right|_{t=0}=u_{0}, \quad \text { in } \Omega, \\
& \partial_{\boldsymbol{n}} u=b(u) \nabla w \cdot \boldsymbol{n}=0, \quad \text { on } \partial \Omega .
\end{aligned}
$$

Our minimal hypotheses on data, which will be kept for the whole of the paper, can be stated as follows:

$$
\begin{aligned}
& b(r)=r^{s}+\beta r^{n}, \quad \beta \geq 0, r \geq 0 ; \\
& 0 \leq n \leq s<10 \text { if } d=3, \quad 0 \leq n \leq s \text { if } d=2 ; \\
& f(r)=-\frac{1}{r^{\kappa}}, \quad \kappa>1, \quad \kappa \geq s+1, r>0 ; \\
& \gamma \in W^{1, \infty}(\mathbb{R}) \cap L^{1}(\mathbb{R}) ; \\
& g \in V \cap L^{\infty}(\Omega), \quad \hat{g}:=\|g\|_{L^{\infty}(\Omega)} ; \\
& \delta \geq 0 .
\end{aligned}
$$

Assumptions (1.5)-(1.6) prescribe that the mobility function $b(u)$ may degenerate at 0 as a power of $u$. If $\beta>0$, we are admitting the growth rate of $b$ at infinity

Received by the editors September 6, 2010 and, in revised form, November 15, 2011. 2010 Mathematics Subject Classification. Primary 35K35, 35K65, 37L30. 
to be faster than its degeneration rate at 0 . For most purposes, the case $\beta>0$ is simpler, because most mathematical difficulties arise from the degeneration of $\beta$ at 0 and not from its growth at $\infty$. Nonetheless, taking some upper bound of the growth of $b$ (i.e. the assumption $s<10$ ) seems to be needed in order to control the mixed term $b(u) \nabla w$ in (1.1).

The sum $f+\gamma$ stands for the derivative of a configuration potential $W$. In particular, $f$ is the (dominating) monotone part and $\gamma$ represents a smooth and globally summable perturbation. Physically, the singular behavior of $f$ at 0 accounts for the presence of short-range repulsive forces, while the perturbation $\gamma$ is related to the occurrence of long-range attractive forces. Note that, globally, $W$ is allowed to present a nonconvex behavior. Finally, we admit the presence of a viscosity effect in (1.2), occurring in case $\delta>0$, as well as of an external forcing term $g$.

In the two-dimensional case, problem (1.1)-(1.4) can describe the evolution of some classes of thin liquid films, with $u$ representing the height of the film. An extensive presentation of the underlying physical situation is given in [8, 32 to which we refer for more details (see also [5, 25] and Remark 2.2 below).

In the three-dimensional case, our system is also physically relevant due to its connections with the Cahn-Hilliard model. Indeed, if \pm 1 represent the pure states of the order parameter in the Cahn-Hilliard model, then we can modify $f$ into the form $f(u) \sim\left(1-u^{2}\right)^{-\kappa}$ and accordingly suppose that $b$ degenerates near \pm 1 (instead of near 0$)$ as a power of $\left(1-u^{2}\right)$. Up to these modifications, system (1.1)-(1.2) turns out to represent a variant of the Cahn-Hilliard model with degenerate mobility and singular potentials analyzed in a large number of papers (cf., e.g., 3 , 4, 20, 29, 31, 33. and the references therein). Correspondingly, all the results proved here under assumptions (1.5)-(1.10) also apply to the Cahn-Hilliard reformulation of the system with straighforward modifications in the proofs. To be more precise, in the CahnHilliard setting we would even have slightly stronger results since it would no longer be necessary to take care of the growth of $b$ at infinity (cf. Remark 2.1 below). This also motivates the choice of considering the viscous case $\delta>0$, which is particularly meaningful in the context of Cahn-Hilliard models (cf. [26]; see also [30]).

The mathematical literature on degenerate fourth order parabolic equations is very wide. For this reason, we limit ourselves to recall, with no claim of completeness, some contributions that, from our point of view, are most closely related to the subject of the present paper. We refer, e.g., to [5] for a more detailed review. Existence and qualitative properties of solutions to the thin-film equation without forcing terms and zero viscosity (i.e., (1.1)-(1.2) with $f, \gamma, g$ and $\delta$ set equal to 0) have been addressed in 6, 7] (in the 1D setting) and in [16, 22, (in the 2-3D setting). The case with a nonzero (and generally power-like) $f(u)$ has been treated as well (see, e.g., 9, 10 for the 1D case and [17 for the 2-3D case). In these papers, existence is proved under various types of regularity assumptions on the initial data and on the nonlinear terms. Moreover, important qualitative properties of solutions like positivity, self-similar behavior, and finite (or infinite) speed of propagation are discussed in a number of significant cases. In particular, these works provide the basic tools which are also at the core of our existence proof, i.e., the energy and entropy bounds that characterize the evolutionary process.

Models very similar to ours are treated in [8, 25, 32] (1D setting) and [24] (2$3 \mathrm{D})$, where the case with both $f$ and $\gamma$ different from 0 is considered. In the papers [24, 25] problem (1.1)-(1.4) is addressed under hypotheses on the nonlinear terms 
very close to ours, the main difference being represented by the fact that we can take more general assumptions on the mobility (namely, we admit $s<10$, the analysis in 24] being restricted to the case $s \in(0,4)$, at least in the 3D case). On the other hand, we have here the restriction $\kappa \geq s+1$ which was not assumed in [24]. A physical motivation for this choice comes from the model example (cf., e.g., [8] or 24])

$$
\begin{aligned}
& b(u)=u^{3}+\beta^{3-n} u^{n}, \quad n \in(0,3), \\
& f(u)=A u^{-3}-B u^{-9} \quad \text { or } f(u)=A u^{-3}-B u^{-4},
\end{aligned}
$$

where $A, B, \beta>0$. Mathematically, the choice of a large $\kappa$ allows us not only to simplify the proof of the existence of a weak solution, but also to use the Moser-type iteration technique for improving the regularity of the constructed weak solution in the viscous case. However, we have to point out that assumption $\kappa \geq s+1$ is not satisfied for other classes of physically relevant models (see, e.g., [32]).

The existence proof given in [24, 25] is based on a nonnegativity-preserving finite-element scheme, which is also effective for a numerical investigation for the model. The paper [8] analyzes the properties of the stationary problem associated to (1.1)-(1.4) in the one-dimensional setting and, in particular, describes the stability properties of the steady states. Finally, the very recent contribution [32] discusses existence and fine qualitative properties of one-dimensional solutions, considering in particular the case, complementary to ours, where $\kappa<1$.

Although our investigation strongly relies on the same basic energy and entropy estimates used in most of the quoted papers, here we address problem (1.1)-(1.4) under a different perspective and, for this reason, we need to manage solutions in a somehow different manner. Actually, we are mostly interested in analyzing the long-time behavior of solutions from the point of view of infinite-dimensional dynamical systems, trying to determine the existence of a global attractor. This object describes the asymptotic behavior of bundles of solutions originating from a generic set $B$ of initial data that is bounded in the natural sense prescribed by the existence theorem (here meaning that the initial energy and entropy have to be uniformly controlled in $B$ ). In a sense, knowing the qualitative properties of a single solution gives no help in this analysis, since the global attractor, describing the behavior of many trajectories, can have a very complicated structure, especially in cases, such as this, where very weak asymptotic compactness properties are expected to hold. In addition to that, it is also worth noticing that many geometric properties holding for solutions of the "homogeneous" system are here "destroyed" by the presence of the external forcing term $g$. For these reasons, here our attention is mostly devoted to determining the (widest) class of solutions that satisfy the energy and entropy inequalities (or, possibly, equalities), that are at the core of the proof of existence for the attractor.

Actually, the literature devoted to the long-time analysis of the Cahn-Hilliard equation with constant mobility from the attractors' perspective is very wide. This starts from the pioneering works [18, 21] (see also Temam's book 34]) and arrives at very sophisticated recent contributions such as 29 . On the contrary, at least to our knowledge, only two papers have been devoted to the analysis of the case of a nonconstant $b(u)$. In [33, the long-time behavior of the Cahn-Hilliard equation with nondegenerate mobility has been addressed under the same assumptions that guarantee existence (see [3]). Then, in the recent work [35, the long-time behavior 
of a degenerate parabolic system very close to (1.1)-(1.2) is studied in the onedimensional setting. In this situation, the authors can prove a strict positivity property also in the nonviscous case, which allows them to show existence of a smooth global attractor by relying on the standard theory of infinite-dimensional dynamical systems.

Coming to our results, our first purpose in this paper is to prove existence of at least one weak solution to problem (1.1)-(1.4) complemented with the proper initial condition. The proof of this result relies on a regularization, a priori estimates, passage to the limit scheme similar to the procedure originally devised in [20] for the Cahn-Hilliard equation. With respect to the proof given in [20], here we have the additional difficulty that in the thin film setting $u$ is not a priori bounded. Hence, we have some regularity loss (cf. (2.13) below) due to fast growth of $b(u)$ at infinity. As a further difference, in 20] the case (in our notation) $\kappa \leq s-1$ is addressed; in other words, differently from here, the degeneration of $b$ dominates the singular character of $f$.

Comparing our proof with that given in 24. for a system very similar to ours, we do not have strict positivity of approximate solutions here. Nevertheless, as a by-product of our procedure, we see that the possibly singular solutions to (1.1)(1.4) can be approximated by the smooth and positive solutions of a regular PDE. Actually, if we consider, for instance, the nonviscous equation (i.e., the case $\delta=$ 0 ), then we can construct the $\varepsilon$-approximation taking $\delta_{\varepsilon}>0$ (which, as noted above, is physically motivated at least in the Cahn-Hilliard setting) and choosing a sufficiently singular function $f_{\varepsilon}$, with $\delta_{\varepsilon} \searrow 0$ and $f_{\varepsilon} \rightarrow f$ in the limit $\varepsilon \searrow 0$. Then, for $\varepsilon>0$, Theorem 6.1 applies (cf. also Remark 6.3); hence, the approximating solutions $u_{\varepsilon}$ are smooth and positive. Moreover, the very same argument used to pass to the limit in Subsection 3.3 below shows that any limit point of $\left\{u_{\varepsilon}\right\}$ for $\varepsilon \searrow 0$ solves the original nonviscous problem.

It is also worth noting that, very probably, using more refined entropy estimate techniques (such as, e.g., in [17]), our existence result could be extended to cover more general assumptions on the nonlinear terms. So, we do not claim optimality of our approach. Actually, the assumptions we deal with have been chosen since they can provide, by a simple proof, existence of a solution in a class which is very flexible and suitable for the long-time analysis. More precisely, our construction is based only on the natural energy and entropy estimates, which can be easily managed to get, for example, dissipativity of the (multivalued) dynamical process associated to the system (cf. Theorem 4.1 below). This in particular entails existence of a (weak) trajectory attractor in the sense of Chepyzhov and Vishik [13.

Our subsequent results regard only the viscous case $\delta>0$ (and in particular can be applied to the Cahn-Hilliard model up to the modifications described above). Then, we can also prove that the trajectory attractor can be intended in the strong sense (i.e., w.r.t. the strong topology of the natural phase space) at least under slightly more restrictive conditions on $f$. The proof relies on an ad-hoc integration by parts formula and a variant of the so-called energy method (cf. [1]; see also [2, 28, and 14, 15, 19] for applications to trajectory attractors). If we further restrict the class of admissible functions $f$ (namely, asking $\kappa$ to be large enough), then we can also prove that weak solutions become strictly separated from 0 for any time $t>0$ (and uniformly for large times). In other words, we can prove that the minimum height of the film is strictly positive, so that the degenerate character 
of the system is in fact lost for positive times. This result, which is in our opinion the main achievement of this paper, is shown by means of a suitable version of the Moser iteration scheme which takes time-regularization effects into account. As a further consequence of this "separation" property, we can also prove arbitrarily high regularity of weak solutions, as well as uniqueness, at least for $t>0$. In turn, this permits us to interpret the global attractor according to the standard theory for single-valued semiflows [1, 34, rather than in the trajectory sense.

The plan of the paper is as follows. In Section 2 we will report our notation and hypotheses and the statement of our existence result. Its proof is divided into several steps and will be presented in Section 3. In Section 4, we will show the existence of weak trajectory attractors. In Section 5, we will prove existence of a strong trajectory attractor in the viscous case $\delta>0$ by applying the so-called energy method. Finally, in Section 6, we will prove the strict positivity of $u$ in the viscous case under more restrictive growth conditions on $f$.

\section{EXISTENCE RESUlT}

Let $\Omega$ be a smooth bounded domain of $\mathbb{R}^{d}, d \in\{2,3\}$. Let $T>0$ be a given final time and set $Q:=\Omega \times(0, T)$. Let $H:=L^{2}(\Omega)$, endowed with the standard scalar product $(\cdot, \cdot)$ and norm $\|\cdot\|$. Also let $V:=H^{1}(\Omega)$. Let $\|\cdot\|_{X}$ denote the norm in the generic Banach space $X$. In this section, we introduce a rigorous variational formulation of the initial-value problem for system (1.1)-(1.4) and state our related existence result holding under conditions (1.5)-1.10). In particular, the assumptions on the nonlinear terms $b, f$ and $\gamma$ deserve some further explanation:

Remark 2.1. Our key assumption is that the singular character of $f$ has to dominate over the degeneracy of $b$ at 0 (cf. (1.7) ). Actually, one could see with straightforward modifications in the proofs that in the case $\beta>0$ (i.e., if $b(r)$ has a lower order degeneration $r^{n}$ at 0 ; cf. (1.5) ), then it would be enough to ask $\kappa \geq n+1$ rather than $\kappa \geq s+1$ in (1.7). We also point out that the requirement $s<10$ in the threedimensional case is motivated by the growth of $b$ at $\infty$ (and not by its degeneration at 0 ). Consequently, in the application to the Cahn-Hilliard model (where solutions have to stay in between the two barriers $r= \pm 1$ ), $s$ could in fact be arbitrary also in the case $d=3$.

We define, whenever they make sense, the following functions:

$$
F(r):=\frac{1}{\kappa-1}+\int_{1}^{r} f(\tau) \mathrm{d} \tau, \quad \Gamma(r):=\int_{1}^{r} \gamma(\tau) \mathrm{d} \tau, \quad W(r):=F(r)+\Gamma(r) .
$$

Remark 2.2. According, e.g., to [8], a physically relevant expression for $W^{\prime}=f+\gamma$ is given by

$$
W^{\prime}(r) \sim-\frac{1}{r^{\kappa}}+\frac{1}{r^{k}}, \quad \text { where } k \in(1, \kappa) .
$$

Actually, this situation is not covered by our assumptions (1.7)-(1.8). However, it is clear that, just with technical modifications in the proofs, one could replace (1.7) with something like

$$
c_{1} \frac{1}{r^{\kappa+1}} \leq f^{\prime}(r) \leq c_{2} \frac{1}{r^{\kappa+1}}
$$


for all $r>0$ and some $c_{1}, c_{2}>0$. Assuming (2.3) and properly choosing $\gamma$, it is clear that we can deal with the case (2.2). Nonetheless, we will assume (1.7) in place of (2.3) in order to reduce technical complications in the proofs.

Next, we introduce the energy functional associated to system (1.1)-(1.2):

$$
\mathcal{E}(u):=\int_{\Omega}\left(\frac{|\nabla u|^{2}}{2}+W(u)-g u\right) .
$$

This leads to defining the energy space, which will act as a phase space for our system:

$$
\mathcal{X}:=\left\{u \in V: u \geq 0 \text { a.e., } u^{1-\kappa} \in L^{1}(\Omega)\right\} .
$$

The space $\mathcal{X}$ is endowed with the natural (graph) metric

$$
\mathrm{d}_{\mathcal{X}}\left(u_{1}, u_{2}\right):=\left\|u_{1}-u_{2}\right\|_{V}+\left\|u_{1}^{1-\kappa}-u_{2}^{1-\kappa}\right\|_{L^{1}(\Omega)},
$$

which is readily proved to be complete. Given $\mu \in(0, \infty)$, we also define

$$
\mathcal{X}_{\mu}:=\left\{u \in \mathcal{X}: u_{\Omega}=\mu\right\},
$$

$(\cdot)_{\Omega}$ denoting here and below the spatial average over $\Omega$. Actually, integrating (1.1) in space one easily sees that the quantity $u_{\Omega}(t)$ is conserved in time for any solution $u$.

Thanks to (1.8), (1.9) and to the above conservation property, a direct computation shows that, for some $\alpha_{\mu}, c_{\mu}, C_{\mu}>0$ (also depending on $g$ ), we have

$$
\alpha_{\mu}\left(\|u\|_{V}^{2}+\left\|u^{1-\kappa}\right\|_{L^{1}(\Omega)}\right)-C_{\mu} \leq \mathcal{E}(u) \leq c_{\mu}\left(1+\|u\|_{V}^{2}+\left\|u^{1-\kappa}\right\|_{L^{1}(\Omega)}\right)
$$

for all $u \in \mathcal{X}_{\mu}$. In particular, for a function $u$ of assigned spatial mean $\mu$, the finiteness of the energy $\mathcal{E}(u)$ corresponds exactly to the condition $u \in \mathcal{X}_{\mu}$.

The above notation is sufficient to define the class of weak solutions.

Definition 2.3. A weak solution to problem (1.1)-(1.4) is a couple $(u, w)$, with

$$
\begin{aligned}
& u \in L^{\infty}(0, T ; V) \cap L^{2}\left(0, T ; H^{2}(\Omega)\right), \quad \delta^{1 / 2} u \in H^{1}(0, T ; H), \\
& F(u) \in L^{\infty}\left(0, T ; L^{1}(\Omega)\right), \\
& w \in L^{5 / 4}\left(0, T ; W^{1,5 / 4}(\Omega)\right), \\
& b^{1 / 2}(u) \nabla w \in L^{2}(0, T ; H), \\
& b(u) \nabla w \in L^{\frac{20}{10+s}}(Q), \quad u_{t} \in L^{\frac{20}{10+s}}\left(0, T ;\left(W^{1, \frac{20}{10-s}}\right)^{*}(\Omega)\right), \\
& f(u) \in L^{2}\left(0, T ; L^{1}(\Omega)\right) \cap L^{5 / 3}(Q)
\end{aligned}
$$

such that the following relations hold a.e. in $(0, T)$ :

$$
\begin{aligned}
& \left\langle u_{t}, \phi\right\rangle+\langle b(u) \nabla w, \nabla \phi\rangle=0 \quad \forall \phi \in W^{1, \frac{20}{10-s}}(\Omega), \\
& \langle w, \psi\rangle=\delta\left(u_{t}, \psi\right)+\langle\nabla u, \nabla \psi\rangle+\langle f(u)+\gamma(u)-g, \psi\rangle \quad \forall \psi \in V,
\end{aligned}
$$

where $\langle\cdot, \cdot\rangle$ denote suitable duality pairings, and such that, in addition,

$$
\left.u\right|_{t=0}=u_{0}, \quad \text { a.e. in } \Omega \text {. }
$$

Remark 2.4. The above regularity framework actually refers to the three-dimensional case. In particular, the "strange" spaces in (2.13) come out due to the necessity of estimating the product term $b(u) \nabla w$ in the presence of a (possibly) fast growing mobility $b$. If $d=2$, we expect that regularity properties stronger 
than (2.11)-(2.14) could be shown to hold (we leave the details to the reader). It is also likely that, for $\delta=0$, (2.11)-(2.14) could be improved by using more refined entropy estimates (as, e.g., in [17]). However, it is not clear whether these techniques could be adapted to the viscous case. Moreover, the resulting class of solutions would probably be less flexible for the purposes of the long-time analysis we aim to perform.

We can now state our basic existence result.

Theorem 2.5. Let us assume (1.5)-(1.10) and let, for some $\mu \in(0, \infty)$,

$$
u_{0} \in \mathcal{X}_{\mu} .
$$

Then, problem (1.1)-(1.4) admits at least one weak solution.

\section{Proof of Theorem 2.5}

We will give the proof in the case $d=3$ and just point out some minor differences occurring in the two-dimensional case.

3.1. Regularized problem. First of all, we introduce a suitably approximated statement. Namely, given $\varepsilon \in(0,1)$, we set

$$
\begin{aligned}
& b_{\varepsilon}(r):=b\left(\left(r^{2}+\varepsilon^{a}\right)^{1 / 2}\right), \\
& f_{\varepsilon}(r):= \begin{cases}f(r) & \text { if } r \geq \varepsilon, \\
f(\varepsilon)+f^{\prime}(\varepsilon)(r-\varepsilon)=-\frac{1}{\varepsilon^{\kappa}}+\frac{\kappa}{\varepsilon^{\kappa+1}}(r-\varepsilon) & \text { if } r<\varepsilon,\end{cases}
\end{aligned}
$$

where $a>0$ will be chosen later on, and it is intended that $f_{\varepsilon}$ is defined for all $r \in \mathbb{R}$. It is then worth noting that

$$
f_{\varepsilon}^{\prime}(r):= \begin{cases}\frac{\kappa}{r^{\kappa+1}} & \text { if } r \geq \varepsilon, \\ \frac{\kappa}{\varepsilon^{\kappa+1}} & \text { if } r<\varepsilon .\end{cases}
$$

Moreover, setting

$$
F_{\varepsilon}(r):=\frac{1}{\kappa-1}+\int_{1}^{r} f_{\varepsilon}(\tau) \mathrm{d} \tau
$$

we obtain that

$$
F_{\varepsilon}(r):= \begin{cases}\frac{1}{(\kappa-1) r^{\kappa-1}} & \text { if } r \geq \varepsilon, \\ \frac{1}{(\kappa-1) \varepsilon^{\kappa-1}}-\frac{1}{\varepsilon^{\kappa}}(r-\varepsilon)+\frac{\kappa}{2 \varepsilon^{\kappa+1}}(r-\varepsilon)^{2} & \text { if } r<\varepsilon .\end{cases}
$$

At this point, we can consider the approximate statement

$$
\begin{aligned}
& u_{\varepsilon, t}-\operatorname{div}\left(b_{\varepsilon}\left(u_{\varepsilon}\right) \nabla w_{\varepsilon}\right)=0, \\
& w_{\varepsilon}=\delta u_{\varepsilon, t}-\Delta u_{\varepsilon}+f_{\varepsilon}\left(u_{\varepsilon}\right)+\gamma\left(u_{\varepsilon}\right)-g,
\end{aligned}
$$

coupled with the initial conditions and the no-flux boundary conditions. Then, in analogy with [20, Thms. 2 and 4] (see also [3, Thm. 2.1]), we have the following existence result for approximate solutions. 
Theorem 3.1. Let us assume (1.5)-(1.10) and let $b_{\varepsilon}, f_{\varepsilon}$ be specified by (3.1)-(3.2). Let, in addition, $u_{0, \varepsilon} \in H^{3}(\Omega)$ be defined as the (unique) solution to the elliptic problem

$$
u_{0, \varepsilon}-\varepsilon^{2} \Delta u_{0, \varepsilon}=u_{0},\left.\quad \partial_{\boldsymbol{n}} u_{0, \varepsilon}\right|_{\partial \Omega}=0 .
$$

Then, there exists at least one couple $\left(u_{\varepsilon}, w_{\varepsilon}\right)$ with

$$
u_{\varepsilon} \in L^{\infty}(0, T ; V) \cap L^{2}\left(0, T ; H^{3}(\Omega)\right), \delta^{1 / 2} u_{\varepsilon} \in H^{1}(0, T ; H), \delta u_{\varepsilon} \in H^{1}(0, T ; V),
$$

$$
w_{\varepsilon} \in L^{2}(0, T ; V),
$$

satisfying (3.6)-(3.7) a.e. in $\Omega \times(0, T)$, together with

$$
\left.u_{\varepsilon}\right|_{t=0}=u_{0, \varepsilon}, \text { a.e. in } \Omega \text {. }
$$

Moreover, in the case $\delta>0$, the couple $\left(u_{\varepsilon}, w_{\varepsilon}\right)$ is unique.

Sketch of proof of Theorem 3.1. We can proceed by following very closely the proofs of the quoted results [20, Thms. 2 and 4], [3, Thm. 2.1]. For this reason, we will just give some very brief highlights. Actually, the main difference here is due to the growth of $b_{\varepsilon}$ at infinity. Nevertheless, one can of course truncate $b_{\varepsilon}$ near $\infty$ and replace it by some approximation $b_{\varepsilon, \nu}$ of at most linear growth, such that $b_{\varepsilon, \nu}$ suitably tends to $b_{\varepsilon}$ as $\nu \searrow 0$. Then, existence for $\nu>0$ is proved similarly as in [20, 3] and it remains to prove suitable a priori estimates uniform w.r.t. $\nu$. However, for the sake of simplicity we will omit the $\nu$-approximation and rather perform formal estimates on the $\varepsilon$-solution in order to show that it fulfills the regularity properties (3.9)-(3.10).

Thus, we can first perform the energy and entropy estimate, as below. Using that $b_{\varepsilon} \geq \varepsilon^{a s / 2}$ and the Lipschitz continuity of $f_{\varepsilon}$, we then obtain

$$
\begin{aligned}
& \left\|u_{\varepsilon}\right\|_{L^{\infty}(0, T ; V) \cap L^{2}\left(0, T ; H^{2}(\Omega)\right)}+\delta^{1 / 2}\left\|u_{\varepsilon}\right\|_{H^{1}(0, T ; H)} \leq c_{\varepsilon}, \\
& \left\|b_{\varepsilon}^{1 / 2} \nabla w_{\varepsilon}\right\|_{L^{2}(0, T ; H)}+\left\|w_{\varepsilon}\right\|_{L^{2}(0, T ; V)} \leq c_{\varepsilon} .
\end{aligned}
$$

Next, we test (3.7) by $\Delta^{2} u_{\varepsilon}$. Using (3.8), (3.12)-(3.13), (1.9) and the global Lipschitz continuity of $f_{\varepsilon}$ and $\gamma$, it is then not difficult to obtain

$$
\left\|u_{\varepsilon}\right\|_{L^{2}\left(0, T ; H^{3}(\Omega)\right)}+\delta^{1 / 2}\left\|u_{\varepsilon}\right\|_{L^{\infty}\left(0, T ; H^{2}(\Omega)\right)} \leq c_{\varepsilon} .
$$

In case $\delta>0$, we can also test (3.7) by $-\Delta u_{\varepsilon, t}$, which yields

$$
\delta\left\|u_{\varepsilon}\right\|_{H^{1}(0, T ; V)} \leq c_{\varepsilon} .
$$

This gives all the desired regularity properties. Finally, to prove uniqueness in the case $\delta>0$, we can test the difference of (3.7) by the difference of the $u_{\varepsilon, t}$ and the difference of (3.6) by the difference of $w_{\varepsilon, t}$. The details are left to the reader.

3.2. A priori estimates. We now aim to obtain a number of a priori bounds, uniform in $\varepsilon$, with the purpose of removing the approximation.

Energy estimate. We test (3.6) by $w_{\varepsilon}$, (3.7) by $u_{\varepsilon, t}$, and sum the results. We then obtain

$$
\frac{\mathrm{d}}{\mathrm{d} t} \mathcal{E}_{\varepsilon}\left(u_{\varepsilon}\right)+\delta\left\|u_{\varepsilon, t}\right\|^{2}+\int_{\Omega} b_{\varepsilon}\left(u_{\varepsilon}\right)\left|\nabla w_{\varepsilon}\right|^{2}=0
$$


where

$$
\mathcal{E}_{\varepsilon}(u):=\int_{\Omega}\left(\frac{|\nabla u|^{2}}{2}+F_{\varepsilon}(u)+\Gamma(u)-g u\right) .
$$

Remark 3.2. We point out that, even at the approximate level, this formal estimate is not completely justified in the case $\delta=0$. Actually, $w_{\varepsilon}$ is (only) in $L^{2}(0, T ; V)$, while (3.6) is not an equation in $L^{2}\left(0, T ; V^{\prime}\right)$ since $b_{\varepsilon}$ grows fast at infinity. However it is clear that, performing a truncation of $b_{\varepsilon}$ and then passing to the limit, the estimate could be justified.

Then, we integrate (3.16) in time and notice that, by (3.5),

$$
\int_{\Omega} F_{\varepsilon}\left(u_{0, \varepsilon}\right) \leq \int_{\Omega} F\left(u_{0, \varepsilon}\right) \leq \int_{\Omega} F\left(u_{0}\right)+\left(f\left(u_{0, \varepsilon}\right), u_{0, \varepsilon}-u_{0}\right) \leq \int_{\Omega} F\left(u_{0}\right),
$$

the latter inequality following from (3.8) and Green's formula. Thus, owing to (2.18), (3.16) gives

$$
\begin{aligned}
& \left\|u_{\varepsilon}\right\|_{L^{\infty}(0, T ; V)}+\delta^{1 / 2}\left\|u_{\varepsilon, t}\right\|_{L^{2}(0, T ; H)} \leq c, \\
& \left\|F_{\varepsilon}\left(u_{\varepsilon}\right)\right\|_{L^{\infty}\left(0, T ; L^{1}(\Omega)\right)} \leq c \\
& \left\|b_{\varepsilon}^{1 / 2}\left(u_{\varepsilon}\right) \nabla w_{\varepsilon}\right\|_{L^{2}(0, T ; H)} \leq c .
\end{aligned}
$$

Here and below, $c$ denotes a positive constant, independent of $\varepsilon$ and of time, whose value may vary even inside a single row. We will use the letter $\alpha$ to denote constants used in estimates from below.

Entropy estimate. Let us define, for $r \in(0, \infty)$, the entropy $M$, by setting

$$
m(r):=\int_{1}^{r} \frac{\mathrm{d} \tau}{b(\tau)}, \quad M(r):=\int_{1}^{r} m(\tau) \mathrm{d} \tau .
$$

Clearly, $M$ is a convex function that grows at most like $r$ for $r \sim \infty$. Moreover, let us introduce its approximate version by taking, for $r \in \mathbb{R}$,

$$
m_{\varepsilon}(r):=\int_{1}^{r} \frac{\mathrm{d} \tau}{b_{\varepsilon}(\tau)}, \quad M_{\varepsilon}(r):=\int_{1}^{r} m_{\varepsilon}(\tau) \mathrm{d} \tau .
$$

Clearly, $M_{\varepsilon}$ is a convex function such that $M_{\varepsilon} \leq M$ a.e. in $(0, \infty)$. Moreover, $m_{\varepsilon}$, $M_{\varepsilon}$ tend to $m, M$, respectively, uniformly on compact sets of $(0, \infty)$.

Then, we can test (3.6) by $m_{\varepsilon}\left(u_{\varepsilon}\right)$, (3.7) by $-\Delta u_{\varepsilon}$, and sum the results. We deduce

$\frac{\mathrm{d}}{\mathrm{d} t}\left(\int_{\Omega} M_{\varepsilon}\left(u_{\varepsilon}\right)+\frac{\delta}{2}\left\|\nabla u_{\varepsilon}\right\|^{2}\right)+\left\|\Delta u_{\varepsilon}\right\|^{2}+\int_{\Omega}\left(f_{\varepsilon}^{\prime}\left(u_{\varepsilon}\right)+\gamma^{\prime}\left(u_{\varepsilon}\right)\right)\left|\nabla u_{\varepsilon}\right|^{2}+\left(g, \Delta u_{\varepsilon}\right)=0$, and we have to control some terms. First, by (1.8) and Hölder's inequality, we have

$$
\left.\left|\int_{\Omega} \gamma^{\prime}\left(u_{\varepsilon}\right)\right| \nabla u_{\varepsilon}\right|^{2}+\left(g, \Delta u_{\varepsilon}\right) \mid \leq c\left(1+\left\|\nabla u_{\varepsilon}\right\|^{2}\right)+\frac{1}{2}\left\|\Delta u_{\varepsilon}\right\|^{2} .
$$

We now observe that, for all $\varepsilon \in(0,1), M_{\varepsilon}\left(u_{0, \varepsilon}\right) \leq M\left(u_{0}\right)$ (to prove this, proceed as in (3.18) ). Moreover, we have that $M\left(u_{0}\right) \in L^{1}(\Omega)$. Actually, (2.18) entails $u_{0}^{1-\kappa} \in L^{1}(\Omega)$ and $M(r)$ grows no faster than $r^{2-s}$ in the neighbourhood of 0 , 
which is good since we assumed $\kappa \geq s+1$ (cf. (1.7)). Thus, integrating (3.25) in time we arrive at

$$
\begin{aligned}
& \left\|u_{\varepsilon}\right\|_{L^{2}\left(0, T ; H^{2}(\Omega)\right)} \leq c, \\
& \int_{0}^{T} \int_{\Omega} f_{\varepsilon}^{\prime}\left(u_{\varepsilon}\right)\left|\nabla u_{\varepsilon}\right|^{2} \leq c .
\end{aligned}
$$

Control of the nonlinear terms. Here, our aim is to derive $\varepsilon$-uniform bounds in order to pass to the limit in the terms $b_{\varepsilon}\left(u_{\varepsilon}\right)$ and $f_{\varepsilon}\left(u_{\varepsilon}\right)$.

First of all, by (3.19), (3.26) and interpolation, we obtain, if $d=3$,

$$
\left\|u_{\varepsilon}\right\|_{L^{10}(\Omega \times(0, T))} \leq c,
$$

whereas for $d=2$ we have instead

$$
\left\|u_{\varepsilon}\right\|_{L^{v}(\Omega \times(0, T))} \leq c_{v} \quad \forall v \in[1, \infty) .
$$

Now let us set

$$
\begin{aligned}
& Q_{\varepsilon}^{1}:=\left\{(x, t) \in Q: u_{\varepsilon}(x, t)<\varepsilon\right\}, \\
& Q_{\varepsilon}^{2}:=\left\{(x, t) \in Q: \varepsilon \leq u_{\varepsilon}(x, t) \leq 1\right\}, \\
& Q_{\varepsilon}^{3}:=\left\{(x, t) \in Q: u_{\varepsilon}(x, t)>1\right\}
\end{aligned}
$$

and notice that, by (3.5) and (3.20),

$$
\left|\Omega_{\varepsilon}^{1}(t)\right| \leq c \varepsilon^{\kappa-1} \quad \text { for a.e. } t \in(0, T) .
$$

Here and below, $\Omega_{\varepsilon}^{i}(t), i \in\{1,2,3\}$, is the section of $Q_{\varepsilon}^{i}$ at the generic time $t \in$ $(0, T)$. Also, it is obvious that

$$
\left\|f_{\varepsilon}\left(u_{\varepsilon}\right)\right\|_{L^{\infty}\left(Q_{\varepsilon}^{3}\right)} \leq c .
$$

Next, we remark (cf. (3.1)) that

$$
\frac{1}{b_{\varepsilon}(r)} \leq \begin{cases}\frac{c}{r^{s}+\beta r^{n}} & \text { if } r \geq \varepsilon, \\ \frac{c}{\varepsilon^{a s / 2}} & \text { if } r<\varepsilon .\end{cases}
$$

Hence, using (3.5), (3.20), (3.33) and the condition $\kappa \geq s+1$ (cf. (1.7)), taking $a$ small enough in the definition (3.1) of $b_{\varepsilon}$ we obtain

$$
\left\|b_{\varepsilon}^{-1}\left(u_{\varepsilon}\right)\right\|_{L^{\infty}\left(0, T ; L^{1}(\Omega)\right)} \leq c .
$$

Now setting

$$
z_{\varepsilon}(x, t):=\max \left\{u_{\varepsilon}(x, t), \varepsilon\right\}, \quad v_{\varepsilon}(x, t):=z_{\varepsilon}(x, t)^{\frac{1-\kappa}{2}},
$$

for a.e. $t \in(0, t)$ we have

$$
\begin{aligned}
\left\|v_{\varepsilon}(t)\right\|_{L^{6}(\Omega)}^{2} & \leq c\left(\left\|v_{\varepsilon}(t)\right\|^{2}+\left\|\nabla v_{\varepsilon}(t)\right\|^{2}\right) \\
& \leq c \int_{\Omega_{\varepsilon}^{1}(t)} \frac{1}{\varepsilon^{\kappa-1}}+c \int_{\Omega_{\varepsilon}^{2}(t) \cup \Omega_{\varepsilon}^{3}(t)} \frac{1}{u_{\varepsilon}^{\kappa-1}}+c \int_{\Omega_{\varepsilon}^{2}(t) \cup \Omega_{\varepsilon}^{3}(t)} \frac{\left|\nabla u_{\varepsilon}\right|^{2}}{u_{\varepsilon}^{\kappa+1}}
\end{aligned}
$$

(here and below, we compute the exponents referring to the case $d=3$; for $d=2$, they can be improved, of course). Hence, using (3.20), (3.24) and (3.33), and integrating in time, it is not difficult to arrive at

$$
\left\|v_{\varepsilon}\right\|_{L^{2}\left(0, T ; L^{6}(\Omega)\right)} \leq c,
$$


whence, recalling (3.35) and possibly choosing a smaller $a$,

$$
\left\|b_{\varepsilon}^{-1}\left(u_{\varepsilon}\right)\right\|_{L^{1}\left(0, T ; L^{3}(\Omega)\right)} \leq c .
$$

Then, using (3.21) and either (3.36) or (3.40), we also have

$$
\left\|\nabla w_{\varepsilon}\right\|_{L^{2}\left(0, T ; L^{1}(\Omega)\right)}+\left\|\nabla w_{\varepsilon}\right\|_{L^{1}\left(0, T ; L^{3 / 2}(\Omega)\right)} \leq c,
$$

whence, by interpolation,

$$
\left\|\nabla w_{\varepsilon}\right\|_{L^{5 / 4}(Q)}+\left\|\nabla w_{\varepsilon}\right\|_{L^{5 / 3}\left(0, T ; L^{15 / 14}(\Omega)\right)} \leq c .
$$

We are now ready to give an estimate of the term $f_{\varepsilon}\left(u_{\varepsilon}\right)$. To do this, we first test (3.7) by $u_{\varepsilon}-\mu$, to obtain

$\mu \int_{\Omega}\left|f_{\varepsilon}\left(u_{\varepsilon}\right)\right|=\int_{\Omega}\left(-\delta u_{\varepsilon, t}+\Delta u_{\varepsilon}-\gamma\left(u_{\varepsilon}\right)+g\right)\left(u_{\varepsilon}-\mu\right)+\int_{\Omega} w_{\varepsilon}\left(u_{\varepsilon}-\mu\right)-\int_{\Omega} f_{\varepsilon}\left(u_{\varepsilon}\right) u_{\varepsilon}$ and the terms on the right-hand side are treated as follows:

$$
\begin{aligned}
& \int_{\Omega}\left(-\delta u_{\varepsilon, t}+\Delta u_{\varepsilon}-\gamma\left(u_{\varepsilon}\right)+g\right)\left(\mu-u_{\varepsilon}\right) \leq c\left\|-\delta u_{\varepsilon, t}+\Delta u_{\varepsilon}-\gamma\left(u_{\varepsilon}\right)+g\right\|\left\|\mu-u_{\varepsilon}\right\| \\
& \quad \leq c\left\|-\delta u_{\varepsilon, t}+\Delta u_{\varepsilon}-\gamma\left(u_{\varepsilon}\right)+g\right\|=: \eta_{1},
\end{aligned}
$$

thanks to (3.19), where $\left\|\eta_{1}\right\|_{L^{2}(0, T)} \leq c$ by (1.8), (1.9) and (3.26). Next,

$$
\int_{\Omega} w_{\varepsilon}\left(u_{\varepsilon}-\mu\right)=\int_{\Omega}\left(w_{\varepsilon}-\left(w_{\varepsilon}\right)_{\Omega}\right)\left(\mu-u_{\varepsilon}\right) \leq c\left\|\nabla w_{\varepsilon}\right\|=: \eta_{2},
$$

with $\left\|\eta_{2}\right\|_{L^{2}(0, T)} \leq c$ by (3.41). Finally, due to (3.2) it is clear that

$$
-\int_{\Omega} f_{\varepsilon}\left(u_{\varepsilon}\right) u_{\varepsilon} \leq \frac{\mu}{2} \int_{\Omega}\left|f_{\varepsilon}\left(u_{\varepsilon}\right)\right|+c_{\mu}
$$

Squaring (3.43), integrating in time, and using (3.44)-(3.46), we easily arrive at

$$
\left\|f_{\varepsilon}\left(u_{\varepsilon}\right)\right\|_{L^{2}\left(0, T ; L^{1}(\Omega)\right)} \leq c .
$$

Now setting $\phi_{\varepsilon}:=-\left|f_{\varepsilon}(r)\right|^{2 / 3}$, we test (3.7) by $\phi_{\varepsilon}\left(u_{\varepsilon}\right)-\left(\phi_{\varepsilon}\left(u_{\varepsilon}\right)\right)_{\Omega}$, and integrate in space and time. This gives

$$
\begin{aligned}
& \int_{0}^{T} \int_{\Omega} f_{\varepsilon}\left(u_{\varepsilon}\right) \phi_{\varepsilon}\left(u_{\varepsilon}\right)+\int_{0}^{T}\left(\delta u_{\varepsilon, t}+\gamma\left(u_{\varepsilon}\right)-g, \phi_{\varepsilon}\left(u_{\varepsilon}\right)-\left(\phi_{\varepsilon}\left(u_{\varepsilon}\right)\right)_{\Omega}\right) \\
& =\int_{0}^{T}\left(w_{\varepsilon}-\left(w_{\varepsilon}\right)_{\Omega}, \phi_{\varepsilon}\left(u_{\varepsilon}\right)\right)+\int_{0}^{T}\left(\left(\phi_{\varepsilon}\left(u_{\varepsilon}\right)\right)_{\Omega} \int_{\Omega} f_{\varepsilon}\left(u_{\varepsilon}\right)\right) .
\end{aligned}
$$

Let us first notice that the first term on the left-hand side gives

$$
\int_{0}^{T} \int_{\Omega} f_{\varepsilon}\left(u_{\varepsilon}\right) \phi_{\varepsilon}\left(u_{\varepsilon}\right)=\left\|f_{\varepsilon}\left(u_{\varepsilon}\right)\right\|_{L^{5 / 3}(Q)}^{5 / 3} .
$$

Next, by Hölder's inequality, we obtain

$$
\begin{aligned}
& \left|\int_{0}^{T}\left(\delta u_{\varepsilon, t}+\gamma\left(u_{\varepsilon}\right)-g, \phi_{\varepsilon}\left(u_{\varepsilon}\right)-\left(\phi_{\varepsilon}\left(u_{\varepsilon}\right)\right)_{\Omega}\right)\right| \\
& \quad \leq\left\|\delta u_{\varepsilon, t}+\gamma\left(u_{\varepsilon}\right)+g\right\|_{L^{2}(Q)}^{2}+c\left\|\phi_{\varepsilon}\left(u_{\varepsilon}\right)\right\|_{L^{2}(Q)}^{2} \leq \sigma\left\|f_{\varepsilon}\left(u_{\varepsilon}\right)\right\|_{L^{5 / 3}(Q)}^{5 / 3}+c_{\sigma},
\end{aligned}
$$

where $\sigma$ is a small constant to be chosen below and $c_{\sigma}>0$ depends on $\sigma$. Notice that (3.19), (1.8) and (1.9) have been used here. 
It then remains to control the terms on the right-hand side of (3.48). As far as the first one is concerned, recalling (3.34), we notice that, a.e. in $(0, T)$,

$$
\begin{aligned}
\left(w_{\varepsilon}-\left(w_{\varepsilon}\right)_{\Omega}, \phi_{\varepsilon}\left(u_{\varepsilon}\right)\right) & \leq\left\|\phi_{\varepsilon}\left(u_{\varepsilon}\right)\right\|_{L^{5 / 2}(Q)}\left\|w_{\varepsilon}-\left(w_{\varepsilon}\right)_{\Omega}\right\|_{L^{5 / 3}(Q)} \\
& \leq \sigma\left\|f_{\varepsilon}\left(u_{\varepsilon}\right)\right\|_{L^{5 / 3}(\Omega)}^{5 / 3}+c_{\sigma}\left\|\nabla w_{\varepsilon}\right\|_{L^{15 / 14}(\Omega)}^{5 / 3}
\end{aligned}
$$

Finally, let us estimate the latter term in (3.48). Actually, it is clear that

$$
\int_{0}^{T}\left(\phi_{\varepsilon}\left(u_{\varepsilon}\right)\right)_{\Omega} \int_{\Omega} f_{\varepsilon}\left(u_{\varepsilon}\right) \leq c\left\|f_{\varepsilon}\left(u_{\varepsilon}\right)\right\|_{L^{2}\left(0, T ; L^{1}(\Omega)\right)}^{2}+c \leq c
$$

the latter inequality following from (3.47). Now collecting (3.48)-(3.52) and recalling (3.34), we finally arrive at

$$
\left\|f_{\varepsilon}\left(u_{\varepsilon}\right)\right\|_{L^{5 / 3}(Q)} \leq c .
$$

3.3. Passage to the limit. We will just consider, for brevity, the case $d=3$. For simplicity of notation, let us set $\zeta_{\varepsilon}:=b_{\varepsilon}^{1 / 2}\left(u_{\varepsilon}\right) \nabla w_{\varepsilon}$ and let $\|\cdot\|_{p}$ denote the norm in the space $L^{p}(Q)$. Then, by (3.21),

$$
\left\|\zeta_{\varepsilon}\right\|_{2} \leq c .
$$

Moreover, since $s<10$, (3.28) guarantees that

$$
\left\|b_{\varepsilon}\left(u_{\varepsilon}\right) \nabla w_{\varepsilon}\right\|_{q}=\left\|b_{\varepsilon}^{1 / 2}\left(u_{\varepsilon}\right) \zeta_{\varepsilon}\right\|_{q} \leq c \text { for some } q>1 .
$$

Hence, by comparison in (3.6),

$$
\left\|u_{\varepsilon, t}\right\|_{L^{q}\left(0, T ; W^{-1, q}(\Omega)\right)} \leq c \text { for some } q>1
$$

(of course, if $\delta>0$ we have much more, but we want to deal with the most general case here). Consequently, using (3.19), (3.28), the Aubin-Lions lemma, and Lebesgue's theorem,

$$
u_{\varepsilon} \rightarrow u \quad \text { strongly in } L^{q}(\Omega \times(0, T)) \quad \forall q \in[1,10)
$$

(here and below, all convergence relations are to be intended up to the extraction of nonrelabelled subsequences). Let us now notice that

$$
b_{\varepsilon} \rightarrow \bar{b} \quad \text { uniformly on compact subsets of } \mathbb{R},
$$

where $\bar{b}$ denotes the even extension of $b$ to $\mathbb{R}$. Then, by (3.57), $s<10$ in (1.5), and Lebesgue's theorem again, we obtain

$$
b_{\varepsilon}\left(u_{\varepsilon}\right) \rightarrow \bar{b}(u) \text { strongly in } L^{q}(\Omega \times(0, T)) \text { for some } q>1 .
$$

Analogously, by 3.53),

$$
f_{\varepsilon}\left(u_{\varepsilon}\right) \rightarrow f(u) \text { strongly in } L^{q}(\Omega \times(0, T)) \text { for all } q \in[1,5 / 3),
$$

whence, in particular, the limit $u$ is a.e. nonnegative and we can replace $\bar{b}$ with $b$ in (3.59).

Our next aim is to pass to the limit in the product $b_{\varepsilon}\left(u_{\varepsilon}\right) \nabla w_{\varepsilon}$. To do this, we first notice that, by (3.57) and $s<10$,

$$
b_{\varepsilon}\left(u_{\varepsilon}\right)^{1 / 5} \rightarrow b(u)^{1 / 5} \quad \text { strongly in } L^{q}(Q) \text { for some } q>5 .
$$

Thus, also using (3.42) we arrive at

$$
b_{\varepsilon}\left(u_{\varepsilon}\right)^{1 / 5} \nabla w_{\varepsilon} \rightarrow b(u)^{1 / 5} \nabla w \quad \text { weakly in } L^{q}(Q) \text { for some } q>1 .
$$


Next, interpolating between (3.36) and (3.40), we get

$$
\left\|b_{\varepsilon}\left(u_{\varepsilon}\right)^{-1}\right\|_{5 / 3} \leq c .
$$

Using (3.63) and (3.54), we then obtain

$$
\left\|b_{\varepsilon}\left(u_{\varepsilon}\right)^{1 / 5} \nabla w_{\varepsilon}\right\|_{25 / 17} \leq\left\|\zeta_{\varepsilon}\right\|_{2}\left\|b_{\varepsilon}\left(u_{\varepsilon}\right)^{-3 / 10}\right\|_{50 / 9} \leq c .
$$

Hence, by (3.64), (3.61) and (3.62),

$$
b_{\varepsilon}\left(u_{\varepsilon}\right)^{2 / 5} \nabla w_{\varepsilon}=b_{\varepsilon}\left(u_{\varepsilon}\right)^{1 / 5}\left(b_{\varepsilon}\left(u_{\varepsilon}\right)^{1 / 5} \nabla w_{\varepsilon}\right) \rightarrow b(u)^{2 / 5} \nabla w \text { weakly in } L^{25 / 22}(Q) .
$$

Then, writing $b_{\varepsilon}\left(u_{\varepsilon}\right)^{2 / 5} \nabla w_{\varepsilon}$ as $\zeta_{\varepsilon} b_{\varepsilon}\left(u_{\varepsilon}\right)^{-1 / 10}$ and using (3.54) and (3.63) once more, it is clear that the exponent $25 / 22$ in (3.65) can be improved. Thus, iterating the above procedure and using (3.54) once more, it is not difficult to arrive at

$$
\zeta_{\varepsilon} \rightarrow b(u)^{1 / 2} \nabla w \quad \text { weakly in } L^{2}(Q),
$$

whence, using (3.59) once more (and computing explicitly the exponent), we finally obtain

$$
b_{\varepsilon}\left(u_{\varepsilon}\right) \nabla w_{\varepsilon} \rightarrow b(u) \nabla w \text { weakly in } L^{\frac{20}{10+s}}(Q) .
$$

Thus, we can take the limit of all terms in (3.6)-(3.7) and get back (2.15)-(2.16), where the properties required to test functions depend of course on the regularity conditions proved above. Indeed, (2.9)-(2.14) follow as a direct by-product of the procedure. The proof of Theorem 2.5 is concluded.

\section{WEAK TRAJECTORY ATTRACTORS}

In this section, we construct the so-called weak trajectory attractor for problem (1.1)-(1.2). To avoid technicalities, we will limit ourselves to dealing with the (more degenerate) case $\beta=0$, i.e., $b(u)=u^{s}$, for $s \in[0,10)$ (cf. (1.5)).

We start by proving a dissipativity result holding for the weak solutions constructed in the proof of Theorem 2.5 .

Theorem 4.1. Let the assumptions of Theorem 2.5 hold, with $\beta=0$, and let

$$
\mathbb{E}_{0}:=\mathcal{E}\left(u_{0}\right),
$$

which is finite thanks to (2.18). Then, there exist a weak solution $(u, w)$ and a monotone function $Q:[0, \infty) \rightarrow[0, \infty)$ such that

$$
\mathcal{E}(u(t))+\int_{0}^{t}\left(\delta\left\|u_{t}\right\|^{2}+\left\|b^{1 / 2}(u) \nabla w\right\|^{2}\right) \leq Q\left(\mathbb{E}_{0}\right) \quad \forall t \geq 0 .
$$

More precisely, there exists a set $\mathcal{B}_{0}$ bounded with respect to the metric (2.6), such that, for any $\mathrm{d}_{\mathcal{X}}$-bounded set $B \subset \mathcal{X}_{\mu}$, there exists a time $T_{B} \geq 0$ such that for any initial datum $u_{0} \in B$ there exists at least one weak solution $u$ starting from $u_{0}$ and such that $u(t) \in \mathcal{B}_{0}$ for all $t \geq T_{B}$.

Remark 4.2. Notice that the above result does not claim that dissipativity holds in the whole class of weak solutions, but just that from any admissible initial datum there starts (at least) one weak solution in the dissipative class (cf. Remark 4.7 for further considerations). 
Proof. We integrate (3.16) between 0 and an arbitrary $t>0$. This gives the $\varepsilon$ equivalent of (4.2). Then, we take the $\lim$ inf with respect to $\varepsilon \searrow 0$ and use estimates (3.19), (3.57), (3.66), the fact that $F_{\varepsilon}$ converges to $F$ uniformly on compact sets of $(0, \infty)$, Fatou's Lemma, and the lower semicontinuity of norms with respect to weak or weak star convergences (of course, we will get an energy inequality, and not necessarily an equality, in this way). Relation (4.2) is proved.

To show dissipativity, we start by considering the case $\kappa>s+1$. Then, it is convenient to rewrite the energy inequality in the differential form:

$$
\frac{\mathrm{d}}{\mathrm{d} t} \mathcal{E}(u)+\delta\left\|u_{t}\right\|^{2}+\int_{\Omega} b(u)|\nabla w|^{2} \leq 0,
$$

for a.e. $t>0$. We then notice that, setting $z:=u^{-1}$, we have, from (2.8),

$$
\sigma\left(\left\|z^{\kappa-1}\right\|_{L^{1}(\Omega)}+\|\nabla u\|^{2}\right)-c \leq \mathcal{E}(u) \leq \sigma^{-1}\left(\mu\|z\|_{L^{\kappa}(\Omega)}^{\kappa}+\|\nabla u\|^{2}+1\right),
$$

where $\sigma \in(0,1)$ depends in particular on $\mu=u_{\Omega}$.

Now, similarly with (3.43), we test (1.2) by $u-\mu$, obtaining

$$
\begin{aligned}
\|\nabla u\|^{2}+\mu\|z\|_{L^{\kappa}(\Omega)}^{\kappa} \leq & \int_{\Omega}\left(-\delta u_{t}-\gamma(u)+g\right)(u-\mu)+\left\|z^{\kappa-1}\right\|_{L^{1}(\Omega)}+\int_{\Omega} w(u-\mu) \\
\leq & \frac{1}{2}\|\nabla u\|^{2}+c\left(1+\delta^{2}\left\|u_{t}\right\|^{2}\right)+\frac{\mu}{2}\|z\|_{L^{\kappa}(\Omega)}^{\kappa}+c_{\mu} \\
& +c\left\|w-w_{\Omega}\right\|_{L^{3 / 2}(\Omega)}^{2} \\
\leq & \frac{1}{2}\|\nabla u\|^{2}+c\left(1+\delta^{2}\left\|u_{t}\right\|^{2}\right)+\frac{\mu}{2}\|z\|_{L^{\kappa}(\Omega)}^{\kappa}+c_{\mu}+c\|\nabla w\|_{L^{1}(\Omega)}^{2} .
\end{aligned}
$$

The last term can be controlled this way:

$$
\|\nabla w\|_{L^{1}(\Omega)}^{2} \leq c\left\|z^{s}\right\|_{L^{1}(\Omega)} \int_{\Omega} b(u)|\nabla w|^{2} .
$$

Next, since $\kappa>s+1$, using the first inequality in (4.4) and Jensen's inequality, we obtain, for suitable positive constants $\alpha$,

$$
(\mathcal{E}(u))^{\frac{s}{\kappa-1}} \geq \alpha\left\|z^{s}\right\|_{L^{1}(\Omega)} \geq \alpha\left(z_{\Omega}\right)^{s} \geq \alpha\left(u_{\Omega}\right)^{-s}=\alpha \mu^{-s} .
$$

From (4.4)-(4.7), we then obtain, for a suitable $c_{*}>0$,

$$
\mathcal{E}(u) \leq c_{*}\left(1+\delta^{2}\left\|u_{t}\right\|^{2}+(\mathcal{E}(u))^{\frac{s}{\kappa-1}} \int_{\Omega} b(u)|\nabla w|^{2}\right) .
$$

Thus, assuming $\mathcal{E} \geq 1$, which is of course not restrictive, we can divide by $\mathcal{E}^{s /(\kappa-1)}$ to obtain

$$
(\mathcal{E}(u))^{\frac{\kappa-1-s}{\kappa-1}} \leq c_{*}\left(1+\delta^{2}\left\|u_{t}\right\|^{2}+\int_{\Omega} b(u)|\nabla w|^{2}\right) .
$$

Taking the $(1-\epsilon)$-power for $\epsilon \in(0,1)$, summing to (4.3), and applying Young's inequality, we then have, for some $\alpha>0$,

$$
\frac{\mathrm{d}}{\mathrm{d} t} \mathcal{E}(u)+\alpha\left((\mathcal{E}(u))^{\frac{(\kappa-1-s)(1-\epsilon)}{\kappa-1}}+\delta\left\|u_{t}\right\|^{2}+\int_{\Omega} b(u)|\nabla w|^{2}\right) \leq c_{\epsilon},
$$

whence the thesis follows by integrating in time and applying the comparison principle for ODEs. More precisely, we also have a quantitative decay estimate for the energy. 
In the case $\kappa=s+1$, we can say a little bit less, but dissipativity still holds. Actually, we can repeat the above procedure up to (4.8). Then, we notice that (4.3) implies in particular that

$$
\int_{0}^{\infty}\left(\delta\left\|u_{t}\right\|^{2}+\int_{\Omega} b(u)|\nabla w|^{2}\right) \leq \mathbb{E}_{0}<\infty .
$$

Consequently, there exists at least one time $T_{*}$ such that

$$
T_{*} \in\left[0,2 c_{*} \mathbb{E}_{0}\right] \quad \text { and } \delta\left\|u_{t}\left(T_{*}\right)\right\|^{2}+\int_{\Omega} b\left(u\left(T_{*}\right)\right)\left|\nabla w\left(T_{*}\right)\right|^{2} \leq \frac{1}{2 c_{*}} .
$$

Substituting in (4.8), we then have

$$
\mathcal{E}\left(u\left(T_{*}\right)\right) \leq 2 c_{*}+\delta=: C^{*} .
$$

Then, we obtain $\mathcal{E}(u(t)) \leq C^{*}$ for any $t \geq T_{*}$ by simply observing that, by (4.3), $\mathcal{E}$ is nonincreasing.

As a next step (see [13 for more details), we need to rewrite the dissipative estimate in such a way that, on the one hand, we will be able to control all the norms which are necessary to pass to the weak limit in the space of solutions of the problem considered (and verify that the limit function is again a solution) and, on the other hand, be sure that the corresponding trajectory phase space will be translation invariant.

The following lemma improves the dissipative estimate (4.2) by adding the terms controlled by the entropy estimate.

Lemma 4.3. Let the assumptions of Theorem 4.1 hold. Then, there exists a solution $(u, w)$ of problem (1.1)-(1.2) which satisfies the following estimate:

$$
\begin{aligned}
\mathcal{E}(u(T))+\int_{T}^{T+1} & \delta\left\|u_{t}(t)\right\|^{2}+\left\|b^{1 / 2}(u(t)) \nabla w(t)\right\|^{2}+\|\Delta u(t)\|^{2} \\
& +\left(f^{\prime}(u(t)) \nabla u(t), \nabla u(t)\right) \mathrm{d} t \\
\leq & Q(\mathcal{E}(u(0))) e^{-\alpha T}+C_{*}, \quad \forall T \geq 0,
\end{aligned}
$$

where the positive constants $\alpha$ and $C_{*}$ and the monotone function $Q$ are independent of $t$ and of the concrete choice of the solution $u$.

Proof. As a consequence of Theorem4.1, it is clear that (4.14) holds, for suitable $Q$ and $\alpha$, without the last two terms in the integral on the left-hand side. To control these terms, it is sufficient, in the case $\kappa>s+1$, to sum (3.24) to (4.10) in the preceding proof. In the case $\kappa=s+1$, we know that there exists $T_{*}=T_{*}\left(\mathbb{E}_{0}\right)$ such that the energy is smaller than some constant $C^{*}$ independent of the initial data for any $T \geq T_{*}$ (cf. (4.13)). Then, it is sufficient to integrate (3.24) over $(T, T+1)$ for $T \geq T_{*}$ to get

$$
\begin{aligned}
& \int_{T}^{T+1}\|\Delta u(t)\|^{2}+\left(f^{\prime}(u(t)) \nabla u(t), \nabla u(t)\right) \mathrm{d} t \\
& \leq c+\int_{\Omega} M(u(T))+\frac{\delta}{2}\|\nabla u(T)\|^{2} \leq Q(\mathcal{E}(T)) \leq Q\left(C^{*}\right),
\end{aligned}
$$

as desired. Actually, it is clear that the energy controls from above the terms $M(u)$ and $\|\nabla u\|$. Being pedantic, all these estimates should be done on the level of approximations $u_{\varepsilon}$ with passing to the limit after that; we directly performed the estimate on $u$ just for brevity. 
Remark 4.4. Note also that all the norms involved in our definition of a weak solution (see Theorem 2.5) are under control if we assume that the weak solution satisfies (4.14). This fact, which can be verified exactly as in the proof of Theorem 2.5. is crucial in order to be able to pass to the weak limit on the space of weak solutions and establish that the absorbing set for the trajectory semigroup is indeed closed; see below.

We are now able to define the trajectory phase space and trajectory dynamical system associated with problem (1.1)-(1.2).

Definition 4.5. Let $\mathcal{K}_{+} \subset L^{\infty}\left(\mathbb{R}_{+}, \mathcal{E}\right)$ be the set of all solutions $u$ of problem (1.1)-(1.2) belonging to the class (2.9)-(2.14) which satisfy the following analogue of (4.14):

$$
\begin{aligned}
& \mathcal{E}(u(T))+\int_{T}^{T+1} \delta\left\|u_{t}(t)\right\|^{2}+\left\|b^{1 / 2}(u(t)) \nabla w(t)\right\|^{2}+\|\Delta u(t)\|^{2} \\
& \quad+\left(f^{\prime}(u(t)) \nabla u(t), \nabla u(t)\right) \mathrm{d} t \\
& \leq C_{u} e^{-\alpha T}+C_{*}, \quad \forall T \geq 0
\end{aligned}
$$

for some constant $C_{u}$ depending on the solution $u$. Then, the shift semigroup $T(h)$, $h \geq 0$, acts on $\mathcal{K}_{+}$:

$$
T(h): \mathcal{K}_{+} \rightarrow \mathcal{K}_{+}, \quad(T(h) u)(t):=u(t+h) .
$$

We will refer below to $\mathcal{K}_{+}$and $T(h): \mathcal{K}_{+} \rightarrow \mathcal{K}_{+}$as a trajectory phase space and trajectory dynamical system associated with problem (1.1)-(1.2) respectively.

Furthermore, in order to be able to introduce the attractor of the trajectory dynamical system, we need to specify the topology on $\mathcal{K}_{+}$as well as the class of bounded sets.

Definition 4.6. We endow the set $\mathcal{K}_{+}$with the topology induced by the embedding $\mathcal{K}_{+} \subset \Theta_{+}^{\text {weak }}:=\left[L_{\text {loc }}^{\infty}\left(\mathbb{R}_{+}, H^{1}(\Omega)\right) \cap L_{\text {loc }}^{2}\left(\mathbb{R}_{+}, H^{1}(\Omega)\right)\right]^{w^{*}}$, where $w^{*}$ stands for the weak-star topology, and will refer to it as a weak topology on the trajectory phase space $\mathcal{K}_{+}$.

A set $B \subset \mathcal{K}_{+}$will be called bounded if inequality (4.16) holds uniformly with respect to all $u \in B$, i.e., if

$$
C_{B}:=\sup _{u \in B} C_{u}<\infty .
$$

Remark 4.7. As usual (see [13] for the details), under the general assumptions of Theorem 4.1. we know neither the fact that any weak solution of problem (1.1)(1.2) satisfies the energy inequality (4.16) nor that the constant $C_{u}$ in (4.16) can be expressed in terms of $\mathcal{E}(u(0))$. Actually, it may be possible to construct a solution $u$ which satisfies (4.14) for the initial moment $T=0$ only and be unable to verify its analogue for other initial times. By this reason, attempting to replace (4.16) by (4.14) in the definition of the trajectory phase space $\mathcal{K}_{+}$, we lose the translation invariance $T(h) \mathcal{K}_{+} \subset \mathcal{K}_{+}$, which is crucial for the attractors theory. However, as we will see below, under the more restrictive assumptions of Theorem 5.1, the answer to both the questions posed above is positive. So, in that case, every reasonably defined weak solution satisfies (4.14) and the boundedness condition is equivalent to the boundedness of $u(0)$ in the energy space. 
Finally, we are now able to introduce the trajectory attractor for problem (1.1)(1.2).

Definition 4.8. The set $\mathcal{A}^{\text {tr }} \subset \mathcal{K}_{+}$is a (weak) trajectory attractor for problem (1.1)-(1.2) if the following conditions are satisfied:

1) $\mathcal{A}^{\text {tr }}$ is compact in $\Theta_{+}^{\text {weak }}$.

2) It is strictly invariant with respect to the trajectory semigroup: $T(h) \mathcal{A}^{t r}=$ $\mathcal{A}^{t r}$.

3) It attracts the images of all bounded sets of $\mathcal{K}_{+}$as time tends to infinity, i.e., for every bounded subset $B \subset \mathcal{K}_{+}$and every neighborhood $\mathcal{O}\left(\mathcal{A}^{\text {tr }}\right)$ (in the topology of $\left.\Theta_{+}^{\text {weak }}\right)$, there exists a time $T=T(B, \mathcal{O})$ such that

$$
T(h) B \subset \mathcal{O}\left(\mathcal{A}^{\text {tr }}\right),
$$

for all $h \geq T$.

Next, we can state the existence result for the above introduced object.

Theorem 4.9. Let the assumptions of Theorem 4.1 hold. Then, problem (1.1)(1.2) possesses a trajectory attractor $\mathcal{A}^{\text {tr }}$ in the sense of the above definition. Moreover, this attractor is generated by all complete (i.e., defined for all $t \in \mathbb{R}$ ) and bounded trajectories for that system. Namely, we have

$$
\mathcal{A}^{t r}:=\left.\mathcal{K}\right|_{t \geq 0},
$$

where $\mathcal{K} \subset L^{\infty}(\mathbb{R}, \mathcal{E})$ is the set of all solutions of (1.1)-(1.2) which satisfy

$$
\begin{aligned}
\mathcal{E}(u(T)) & +\int_{T}^{T+1} \delta\left\|u_{t}(t)\right\|^{2}+\left\|b^{1 / 2}(u(t)) \nabla w(t)\right\|^{2}+\|\Delta u(t)\|^{2} \\
& +\left(f^{\prime}(u(t)) \nabla u(t), \nabla u(t)\right) \mathrm{d} t \leq C_{*}
\end{aligned}
$$

for all $T \in \mathbb{R}$ and some $C_{*}>0$.

Proof. As usual (see [13]), in order to show the attractor existence, we only need to verify the existence of a compact and bounded absorbing set for the trajectory dynamical system $T(h): \mathcal{K}_{+} \rightarrow \mathcal{K}_{+}$(the continuity of the semigroup in the $\Theta_{+}^{\text {weak }}$ topology is obvious since it is just a translation semigroup). Note that, due to (4.16), the set $\mathcal{B} \subset \mathcal{K}_{+}$of solutions $u$ satisfying

$$
\begin{gathered}
\mathcal{E}(u(T))+\int_{T}^{T+1} \delta\left\|u_{t}(t)\right\|^{2}+\left\|b^{1 / 2}(u(t)) \nabla w(t)\right\|^{2} \\
+\|\Delta u(t)\|^{2}+\left(f^{\prime}(u(t)) \nabla u(t), \nabla u(t)\right) \mathrm{d} t \leq 2 C_{*}
\end{gathered}
$$

for all $T \geq 0$ will be an absorbing set for the semigroup $T(h)$ acting on $\mathcal{K}_{+}$. Obviously, this set is bounded (in the sense of Definition 4.6). Thus, we only need to verify that it is compact in the $\Theta_{+}^{\text {weak }}$ topology.

Indeed, let $\left\{u_{n}\right\} \subset \mathcal{B}$ be a sequence of solutions. Then, due to estimate (4.20), this sequence is precompact in $\Theta_{+}^{\text {weak }}$, so, without loss of generality, we may assume that $u_{n} \rightarrow u \in \Theta_{+}^{\text {weak }}$ in the topology of $\Theta_{+}^{\text {weak }}$ and we only need to verify that the limit function $u$ solves (1.1)-(1.2) and satisfies (4.20) as well.

The proof of this fact repeats almost word for word the proof of the existence Theorem 2.5 and is even a bit simpler since we do not need to consider the regular approximations to $f$ and $b$ (note that the uniform estimate (4.20) allows us to control uniformly all of the norms involved in (2.9)-(2.14)). By this reason, we leave the rigorous proof to the reader. 
Thus, all of the assumptions of the abstract attractor existence theorem are verified and the theorem is proved.

\section{ENERGy EQUALITIES AND STRONG ATTRACTION}

In the viscous case $\delta>0$ and under slightly more restrictive assumptions on the growth of $f$, we can prove that $\mathcal{A}^{t r}$ is in fact a strong trajectory attractor (i.e., it attracts with respect of the strong topology of $\mathcal{X}$ ). This is the object of our next result:

Theorem 5.1. Let assumptions (1.5)-1.10) hold and let, additionally, $\delta>0$ and $\beta=0$. In addition, let

$$
\kappa \geq \frac{3 s}{2}+1 \text { if } d=3 \quad \text { and } \kappa \geq s+1 \text { if } d=2 .
$$

Then, any weak solution of problem (1.1)-(1.2) satisfies the additional regularity properties

$$
w \in L^{2}(0, T ; H), \quad f(u) \in L^{2}(0, T ; H) .
$$

Moreover, a.e. in $(0, \infty)$, the following energy equality holds:

$$
\frac{\mathrm{d}}{\mathrm{d} t} \mathcal{E}(u)+\delta\left\|u_{t}\right\|^{2}+\int_{\Omega} b(u)|\nabla w|^{2}=0,
$$

as well as the following entropy equality (compare with (3.24)):

$$
\frac{\mathrm{d}}{\mathrm{d} t}\left(\int_{\Omega} M(u)+\frac{\delta}{2}\|\nabla u\|^{2}\right)+\|\Delta u\|^{2}+\int_{\Omega}\left(f^{\prime}(u)+\gamma^{\prime}(u)\right)|\nabla u|^{2}+(g, \Delta u)=0 .
$$

Proof. Let us start by proving (5.3) and first deal with the 3D-case. The key step is given by the following integration by parts formula.

Lemma 5.2. Let $b \in L^{p}(\Omega)$ for some $p>1$, with $b \geq 0$ a.e. in $\Omega$. Also let $b^{-1} \in L^{q}(\Omega)$ for some $q>3 / 2$ if $d=3$ (respectively, for some $q>1$ if $d=2$ ). Let $\phi \in H$ and let $w$ be the (unique) solution to the degenerate elliptic problem

$$
-\operatorname{div}(b \nabla w)+w=\phi, \quad \text { in } \Omega, \quad(b \nabla w) \cdot \boldsymbol{n}=0, \quad \text { on } \Gamma .
$$

Then, $b|\nabla w|^{2} \in L^{1}(\Omega)$ and

$$
(-\operatorname{div}(b \nabla w), w)=\int_{\Omega} b|\nabla w|^{2} .
$$

Remark 5.3. As will be clear from the proof, the regularity of $w$ is sufficient to state (5.5) in that "strong" form. In particular, since we have

$$
b \nabla w \in L^{\frac{2 p}{p+1}}(\Omega), \quad \operatorname{div}(b \nabla w) \in H,
$$

a suitable trace theorem (cf., e.g., [11, Thm. 2.7.6]) permits us to interpret the boundary condition in (5.5) in the sense of trace operators as a relation in the space $W^{-\frac{p+1}{2 p}, \frac{2 p}{p+1}}(\Gamma)$.

Proof of Lemma 5.2. Again, we prove the theorem for $d=3$ and just point out some minor differences occurring for $d=2$. Let $A$ be the Laplace operator with 0-Neumann boundary conditions, namely,

$$
A: V \rightarrow V^{\prime}, \quad\langle A v, z\rangle:=\int_{\Omega} \nabla v \cdot \nabla z .
$$


Then, one can see $A+\mathrm{Id}$ as a strictly positive unbounded operator on $H$ and consider fractional powers of it. For $\varepsilon>0$, we let $b_{\varepsilon}:=\max \{b, \varepsilon\}$. We now consider the approximate problem

$$
\varepsilon A^{3} w_{\varepsilon}-\operatorname{div}\left(b_{\varepsilon} \nabla w_{\varepsilon}\right)+w_{\varepsilon}=\phi, \quad\left(b_{\varepsilon} \nabla w_{\varepsilon}\right) \cdot \boldsymbol{n}=0 \quad \text { on } \Gamma .
$$

Then, testing (5.9) by $w_{\varepsilon}$, we obtain

$$
\varepsilon\left\|A^{3 / 2} w_{\varepsilon}\right\|^{2}+\int_{\Omega} b_{\varepsilon}\left|\nabla w_{\varepsilon}\right|^{2}+\left\|w_{\varepsilon}\right\|^{2}=\left(\phi, w_{\varepsilon}\right) .
$$

Thus, for all $\varepsilon>0$, we have that $w_{\varepsilon} \in D\left((A+\mathrm{Id})^{3 / 2}\right) \subset H^{3}(\Omega)$.

From (5.10), we obtain that $w_{\varepsilon}$ is bounded, independently of $\varepsilon$, in $H$. In addition, we have

$$
\left\|\nabla w_{\varepsilon}\right\|_{L^{\frac{2 q}{q+1}(\Omega)}} \leq\left\|b_{\varepsilon}^{1 / 2} \nabla w_{\varepsilon}\right\|\left\|b_{\varepsilon}^{-1 / 2}\right\|_{L^{2 q}(\Omega)} \leq c
$$

and, since $q>3 / 2$, it follows that $2 q /(q+1)>6 / 5$ (respectively, if $d=2$, from $q>1$ we have $2 q /(q+1)>1)$. Thus, by standard compact embedding results, we have, up to a (nonrelabelled) subsequence of $\varepsilon \searrow 0$,

$$
w_{\varepsilon} \rightarrow w \quad \text { weakly in } W^{1, \frac{2 q}{q+1}}(\Omega) \text { and strongly in } H \text {. }
$$

Moreover, since $p>1$, we can write

$$
\left\|b_{\varepsilon} \nabla w_{\varepsilon}\right\|_{L^{\frac{2 p}{p+1}}(\Omega)} \leq\left\|b_{\varepsilon}^{1 / 2} \nabla w_{\varepsilon}\right\|\left\|b_{\varepsilon}^{1 / 2}\right\|_{L^{2 p}(\Omega)} \leq c .
$$

Thus, $b_{\varepsilon} \nabla w_{\varepsilon}$ is bounded in $L^{2 p /(p+1)}(\Omega) \subset D\left((A+\mathrm{Id})^{-1}\right)$ and, consequently,

$$
\left\|-\operatorname{div}\left(b_{\varepsilon} \nabla w_{\varepsilon}\right)\right\|_{D\left((A+\mathrm{Id})^{-3 / 2}\right)} \leq c
$$

and, proceeding similarly with Subsection 3.3 , we can also prove that $b_{\varepsilon} \nabla w_{\varepsilon}$ tends to $b \nabla w$ weakly in $L^{\frac{2 p}{p+1}}(\Omega)$. Moreover, (5.14) tells us that, for any $\varepsilon>0$, equation (5.9) makes sense at least as a relation in $D\left((A+\mathrm{Id})^{-3 / 2}\right)$ (in particular, the obtained regularity $w_{\varepsilon} \in D\left((A+\mathrm{Id})^{3 / 2}\right)$ justifies having used $w_{\varepsilon}$ as a test function in (5.9) ).

Now, the obvious fact that $b_{\varepsilon} \rightarrow b$ strongly in $L^{p}(\Omega)$, the first of (5.12), and Ioffe's theorem (see, e.g., [27]) give

$$
\int_{\Omega} b|\nabla w|^{2} \leq \liminf _{\varepsilon \searrow 0} \int_{\Omega} b_{\varepsilon}\left|\nabla w_{\varepsilon}\right|^{2} .
$$

Thus, using (5.10) and the second part of (5.12), we can go on as follows:

$$
\begin{aligned}
\int_{\Omega} b|\nabla w|^{2} & \leq \lim _{\varepsilon \searrow 0}\left(\phi-w_{\varepsilon}, w_{\varepsilon}\right)+\liminf _{\varepsilon \searrow 0}\left(-\varepsilon\left\|A^{3 / 2} w_{\varepsilon}\right\|^{2}\right) \\
& \leq(\phi-w, w)=(-\operatorname{div}(b \nabla w), w),
\end{aligned}
$$

where (5.5) has been used to deduce the last equality. Thus, to complete the proof, we have to show the inequality converse to (5.16). Now, $b \nabla w \in L^{2 p /(p+1)}(\Omega)$ thanks to (the $\lim \inf$ of) (5.13). Thus, also $-\operatorname{div}(b \nabla w) \in D\left((A+\mathrm{Id})^{-3 / 2}\right)$ so that we can 
test (5.5) by $w_{\varepsilon} \in D\left((A+\mathrm{Id})^{3 / 2}\right)$ and rigorously integrate by parts to obtain

$$
\begin{aligned}
\left(-\operatorname{div}(b \nabla w), w_{\varepsilon}\right) & =\int_{\Omega} b \nabla w \cdot \nabla w_{\varepsilon} \leq \frac{1}{2} \int_{\Omega} b|\nabla w|^{2}+\frac{1}{2} \int_{\Omega} b\left|\nabla w_{\varepsilon}\right|^{2} \\
& \leq \frac{1}{2} \int_{\Omega} b|\nabla w|^{2}+\frac{1}{2} \int_{\Omega} b_{\varepsilon}\left|\nabla w_{\varepsilon}\right|^{2} \\
& \leq \frac{1}{2} \int_{\Omega} b|\nabla w|^{2}-\frac{\varepsilon}{2}\left\|A^{3 / 2} w_{\varepsilon}\right\|^{2}+\frac{1}{2}\left(\phi-w_{\varepsilon}, w_{\varepsilon}\right),
\end{aligned}
$$

where the fact that $b \leq b_{\varepsilon}$ almost everywhere and the equality (5.10) have also been used. Passing to the limit and using the second part of (5.12) and that $-\operatorname{div}(b \nabla w) \in H$ (as it follows by comparison in (5.5)), we then obtain

$$
(-\operatorname{div}(b \nabla w), w) \leq \frac{1}{2} \int_{\Omega} b|\nabla w|^{2}+\frac{1}{2}(\phi-w, w) \leq \frac{1}{2} \int_{\Omega} b|\nabla w|^{2}+\frac{1}{2}(-\operatorname{div}(b \nabla w), w) .
$$

Namely, we obtained the inequality converse to (5.16), whence the thesis.

We now proceed with the proof of Theorem 5.1 and, precisely, of equality (5.3) under the assumption (5.1). To do this, we first prove (5.2) and, with this purpose, we set $z:=u^{-1}$ and observe that equation (1.2) can be rewritten as

$$
\delta z_{t}+z^{2} \Delta z^{-1}+z^{\kappa+2}=-z^{2} w+z^{2} \phi, \quad \text { where } \phi:=\gamma(u)-g
$$

and we notice that

$$
\|\phi\|_{L^{\infty}(\Omega \times(0, T))} \leq C,
$$

thanks to (1.8)-(1.9). Here and below, $C$ denotes a constant possibly depending on the "energy" of the initial data (cf. (2.18) ) and on the choice of $T$, while $c$ is an absolute constant (i.e., it does not depend on the initial data or on $T$ ). By (2.12), we also know that

$$
\left\|u^{s / 2} \nabla w\right\|_{L^{2}(\Omega \times(0, T))} \leq C .
$$

Next, by the first of (2.14), a comparison in (1.2) also gives

$$
\|w\|_{L^{2}\left(0, T ; L^{1}(\Omega)\right)} \leq C .
$$

At this point, we note that, for $d=3$, thanks to (2.10) and (5.1),

$$
\left\|b^{-1 / 2}(u)\right\|_{L^{\infty}\left(0, T ; L^{3}(\Omega)\right)} \leq C .
$$

Hence, combining (5.23) with (5.22) and (2.12), we readily arrive at

$$
\|w\|_{L^{2}(Q)} \leq c\|w\|_{L^{2}\left(0, T ; W^{1,6 / 5}(\Omega)\right)} \leq C,
$$

whence (5.2) follows simply by comparing terms in (1.2) and taking advantage of (2.9)-(2.14). In the case $d=2$, we only have the $L^{\infty}(0, T ; H)$-norm in (5.23), but (5.2) still follows by using the continuous embedding $W^{1,1}(\Omega) \subset H$ in the analogue of (5.24).

We now proceed with the proof of (5.3). Summing together (1.1) and (1.2), we have

$$
-\operatorname{div}(b(u) \nabla w)+w=\phi:=(\delta-1) u_{t}-\Delta u+f(u)+\gamma(u)-g
$$

and it is clear from (1.8)-(1.9), (2.9) and (5.2) that, for any $T>0,\|\phi\|_{L^{2}(0, T ; H)} \leq$ $C_{T}$. 
Moreover, from (1.6) and (the limit of) (3.28) (or (3.29) ), we have that, a.e. in $(0, T), b(u) \in L^{p}(\Omega)$ for a suitable $p>1$ (e.g., if $d=3$, we can take $\left.p=10 / s\right)$. Finally, thanks to (5.2), it is clear that (if $d=3$, the case $d=2$ being analogous), a.e. in $(0, T)$,

$$
\left\|b^{-1}(u)\right\|_{L^{q}(\Omega)} \leq\left\|z^{s}\right\|_{L^{q}(\Omega)} \leq c\left\|z^{\frac{2(\kappa-1)}{3}}\right\|_{L^{q}(\Omega)}+c \leq C,
$$

for a suitable $q>3 / 2$. Hence, $b=b(u)$ and $\phi$ defined in (5.25) satisfy, a.e. in $(0, T)$, the assumptions of Lemma 5.2. Consequently, as we test (1.1) by $w$ and (1.2) by $u_{t}$, we obtain, thanks to (5.6), the energy equality (5.3), as desired.

Finally, we come to the proof of (5.4). From (2.14) and (5.1), we see that at least $m(u) \in L^{2}(Q)$, so, since due to (2.9) $u_{t} \in L^{2}(Q)$ as well, we have

$$
\frac{\mathrm{d}}{\mathrm{d} t}(M(u), 1)=\left(u_{t}, m(u)\right)=(\operatorname{div}(b(u) \nabla w), m(u)),
$$

where the right-hand side is understood as a scalar product in $L^{2}(Q)$. Thus, we need to verify that

$$
(\operatorname{div}(b(u) \nabla w), m(u))=-(b(u) \nabla w, \nabla m(u))=-(\nabla w, \nabla u)=(w, \Delta u),
$$

almost everywhere in time. To this end, we note that, according to (2.9) and the embedding $H^{2}(\Omega) \subset C(\bar{\Omega})$, we have $u(t), b(u(t)) \in C(\bar{\Omega})$ for almost all $t$. Then, keeping in mind that $b^{1 / 2}(u) \nabla w \in L^{2}(Q)$ by (2.12), we conclude that $b(u(t)) \nabla w(t) \in L^{2}(\Omega)$ for almost all $t$.

Thus, we only need to check that $m(u(t)) \in H^{1}(\Omega)$ for almost all $t$. The fact that this function belongs to $L^{2}(\Omega)$ is already verified, so we need that

$$
\nabla m(u(t))=b^{-1}(u(t)) \nabla u(t) \in L^{2}(\Omega) .
$$

Since, due to (2.9), we know that $\nabla u(t) \in L^{6}(\Omega)$ for almost all $t$, it is sufficient to check that $b^{-1}(u(t)) \in L^{3}(\Omega)$. Actually, this follows immediately from the proved fact that $f(u) \in L^{2}(Q)$ (see the proof of the energy equality) and condition (5.1).

Thus, we have verified that, for almost all $t, m(u(t)) \in H^{1}(\Omega)$ and $b(u) \nabla w(t) \in$ $L^{2}(\Omega)$. This justifies the first two equalities in (15.28). Note that the last one is obvious since $w(t) \in L^{2}(\Omega)$ and $\Delta u(t) \in L^{2}(\Omega)$ for almost all $t$. Thus, we have verified that

$$
\frac{\mathrm{d}}{\mathrm{d} t}(M(u(t)), 1)=(w(t), \Delta u(t))
$$

for almost all $t$. Inserting the expression for $w(t)$ from (1.2) into this identity, we end up with the desired entropy equality (5.4). Theorem 5.1 is proved.

Our next task here is to obtain stronger results on the attraction to the above constructed trajectory attractor under the additional assumptions of Theorem 5.1. We start by stating a couple of corollaries that improve the results of Theorems 2.5 and 4.1 and simplify the construction of the trajectory phase space based on the energy and entropy inequalities obtained above.

Corollary 5.4. Let the assumptions of Theorem 5.1 hold. Then, every weak solution of problem (1.1) -(1.2) satisfies the dissipative estimate (4.14) and, therefore, every weak solution automatically satisfies (4.16) with $C_{u}=Q(\mathcal{E}(u(0)))$. Thus, the condition (4.16) in the definition of the trajectory phase space $\mathcal{K}_{+}$can be omitted and we may naturally consider $\mathcal{K}_{+}$just as the set of all weak solutions of problem (1.11)-(1.2). In addition, for every weak solution $u$, we have $u \in C\left([0, T], H^{1}(\Omega)\right)$ and $u^{1-\kappa} \in C\left([0, T], L^{1}(\Omega)\right)$. 
Indeed, we only need the entropy and energy (in)equalities in order to derive the dissipative estimate (4.14). Since these inequalities now hold for every weak solution, we have this estimate for every weak solution as well. The continuity properties stated in the corollary follow immediately from the energy equality.

Corollary 5.5. Let the assumptions of Theorem 5.1 hold. Then, the set $B$ is bounded in $\mathcal{K}_{+}$, in the sense of Definition 4.6, if and only if the set of the initial data $\{u(0), u \in B\}$ is bounded in the energy space $\mathcal{X}_{\mu}$.

We are now able to state our main result on the strong convergence to the trajectory attractor.

Theorem 5.6. Let the assumptions of Theorem 5.1 hold. Then, the trajectory attractor $\mathcal{A}^{\text {tr }}$ of problem (1.1)-(1.2) is compact in $C_{\text {loc }}\left(\mathbb{R}_{+}, \mathcal{X}\right)$ and the attraction property holds in that strong topology as well (recall that the compactness in $C_{\text {loc }}\left(\mathbb{R}_{+}, \mathcal{X}\right)$ means that the u-component of $\mathcal{A}^{\text {tr }}$ is compact in $C_{\text {loc }}\left(\mathbb{R}_{+}, H^{1}(\Omega)\right)$ and the $u^{1-\kappa}$-component is compact in $C_{\text {loc }}\left(\mathbb{R}_{+}, L^{1}(\Omega)\right)$ ).

Proof. Let $\mathcal{B} \subset K_{+}$be the absorbing set introduced in the proof of Theorem 4.9. We claim that the set

$$
\mathcal{B}_{1}:=T(1) \mathcal{B}
$$

is an absorbing set which is compact in the above-mentioned topology (this is clearly enough for the proof of the theorem). Indeed, let $\left\{u_{n}\right\} \subset \mathcal{B}$ be an arbitrary sequence of solutions. Then, since $\mathcal{B}$ is compact in $\Theta_{+}^{\text {weak }}$, we may assume without loss of generality that $u_{n} \rightarrow u$ in $\Theta_{+}^{w e a k}$, where $u$ also solves the problem (1.1)-(1.2). To verify the above-mentioned compactness, we need to check that

$$
u_{n} \rightarrow u \text { in } C\left([1, N], H^{1}(\Omega)\right), \quad u_{n}^{1-\kappa} \rightarrow u^{1-\kappa} \text { in } C\left([1, N], L^{1}(\Omega)\right),
$$

for every $N>1$. Furthermore, without loss of generality, we may check these convergences for $N=2$ only.

To this end, we will use the proved energy equality, which we will rewrite in the following form:

$$
\begin{aligned}
& T\left[\frac{1}{2}\left\|\nabla u_{n}(T)\right\|^{2}+\left(F\left(u_{n}(T)\right), 1\right)+\left(\Gamma\left(u_{n}(T)\right), 1\right)-\left(g, u_{n}(T)\right)\right] \\
& \quad+\int_{0}^{T} \delta t\left\|\partial_{t} u_{n}(t)\right\|^{2}+t\left(b\left(u_{n}(t)\right) \nabla w_{n}(t), \nabla w_{n}(t)\right) \mathrm{d} t \\
& \quad=\int_{0}^{T} \frac{1}{2}\left\|\nabla u_{n}(t)\right\|^{2}+\left(F\left(u_{n}(t)\right), 1\right)+\left(\Gamma\left(u_{n}(t)\right), 1\right)-\left(g, u_{n}(t)\right) \mathrm{d} t
\end{aligned}
$$

where $T \in[1,2]$. Our next task is to pass to the limit $n \nearrow \infty$ in this inequality. First of all, thanks to the energy and entropy estimates and to the Aubin-Lions compactness theorem, we have

$$
u_{n} \rightarrow u \quad \text { in } C_{w}([1,2] ; V), \quad \text { (strongly) in } C([1,2] ; H) \cap L^{2}(1,2 ; V),
$$

and pointwise (a.e.). Thus, using the first convergence above and Fatou's Lemma, we see that

$$
\frac{1}{2}\|\nabla u(T)\|^{2} \leq \liminf _{n \nearrow \infty} \frac{1}{2}\left\|\nabla u_{n}(T)\right\|^{2}, \quad(F(u(T)), 1) \leq \liminf _{n \nearrow \infty}\left(F\left(u_{n}(T)\right), 1\right) .
$$


Next, thanks also to (1.8)-(1.9),

$$
(\Gamma(u), 1)-(g, u)=\lim _{n \nearrow \infty}\left(\Gamma\left(u_{n}\right), 1\right)-\left(g, u_{n}\right) \quad \text { strongly in } C^{0}([1,2]) .
$$

Moreover, thanks to lower semicontinuity of norms w.r.t. weak convergence and to Ioffe's theorem, we also have

$$
\int_{0}^{T} \delta t\left\|\partial_{t} u(t)\right\|^{2} \mathrm{~d} t \leq \liminf _{n \nearrow \infty} \int_{0}^{T} \delta t\left\|\partial_{t} u_{n}(t)\right\|^{2} \mathrm{~d} t
$$

$$
\int_{0}^{T} t(b(u(t)) \nabla w(t), \nabla w(t)) \mathrm{d} t \leq \liminf _{n \nearrow \infty} \int_{0}^{T} t\left(b\left(u_{n}(t)\right) \nabla w_{n}(t), \nabla w_{n}(t)\right) \mathrm{d} t .
$$

Finally, in order to pass to the limit in (5.30), we only need to prove that $F\left(u_{n}\right) \rightarrow$ $F(u)$ strongly in $L^{1}([0,2] \times \Omega)$. Actually, this follows from the uniform $L^{2}$-bound of $f\left(u_{n}\right)$, the pointwise convergence $u_{n} \rightarrow u$ and the generalized Lebesgue theorem. Thus, we can take the supremum limit $n \nearrow \infty$ in (5.30) and obtain the inequality:

$$
\begin{aligned}
& \underset{n \nearrow \infty}{\limsup _{n} T\left[\frac{1}{2}\left\|\nabla u_{n}(T)\right\|^{2}+\left(F\left(u_{n}(T)\right), 1\right)\right]} \\
& \leq-T[(\Gamma(u(T)), 1)-(g, u(T))]-\int_{0}^{T} \delta t\left\|\partial_{t} u(t)\right\|^{2}-t(b(u(t)) \nabla w(t), \nabla w(t)) \mathrm{d} t
\end{aligned}
$$

$$
+\int_{0}^{T} \frac{1}{2}\|\nabla u(t)\|^{2}+(F(u(t)), 1)+(\Gamma(u(t)), 1)-(g, u(t)) \mathrm{d} t .
$$

On the other hand, applying the energy equality in the form (5.30) directly to the limit solution $u$, and comparing with (5.36), we obtain, for all $T \in(1,2)$,

$$
\limsup _{n \nearrow \infty} T\left[\frac{1}{2}\left\|\nabla u_{n}(T)\right\|^{2}+\left(F\left(u_{n}(T)\right), 1\right)\right] \leq T\left[\frac{1}{2}\|\nabla u(T)\|^{2}+(F(u(T)), 1)\right],
$$

whence, recalling (5.32), we infer

$$
\left\|u_{n}(T)\right\|_{V} \rightarrow\|u(T)\|_{V}, \quad\left\|F\left(u_{n}(T)\right)\right\|_{L^{1}(\Omega)} \rightarrow\|F(u(T))\|_{L^{1}(\Omega)} .
$$

This, together with the weak convergence $u_{n}(T) \rightarrow u(T)$ in $V$ (cf. (5.31) ) and $u_{n} \rightarrow u$ almost everywhere, implies the strong convergence

$$
u_{n}(T) \rightarrow u(T) \text { in } V, \quad u_{n}^{1-\kappa}(T) \rightarrow u^{1-\kappa}(T) \text { in } L^{1}(\Omega),
$$

for all $T \in[1,2]$. This gives the strong convergence $u_{n} \rightarrow u$ in $\mathcal{X}$ pointwise in time. The desired uniform convergence (5.29) can be easily obtained using the standard contradiction arguments and applying the energy equality for $u_{n}\left(T_{n}\right)$ instead of $u_{n}(T)$. Theorem 5.6 is proved.

Corollary 5.7. Arguing in a similar way (and also using the entropy equality), one can verify the compactness and strong convergence to the trajectory attractor in all spaces involved in (2.9) $-(2.14)$. 


\section{Separation from singularities and uniqueness}

In this section we prove that, in the viscous case $\delta>0$, if $\kappa$ is large enough, then any weak solution becomes uniformly strictly positive for any $t>0$. This is the object of the following.

Theorem 6.1. Let assumptions (1.5)-(1.10) hold and let, additionally, $\delta>0$ and $\beta=0$. In addition, let

$$
\kappa>2 s+3 \text { if } d=3, \quad \text { and } \kappa>s+1 \geq 2 \text { if } d=2 .
$$

Then, there exists a function $Q ;[0, \infty)^{2} \rightarrow[0, \infty)$, monotone in each of its arguments, such that any weak solution $u$ satisfies, for any $\epsilon>0$, the separation property

$$
\left\|u^{-1}(t)\right\|_{L^{\infty}(\Omega)} \leq Q\left(\mathbb{E}_{0}, \epsilon^{-1}\right) \text { for a.e. } t \geq \epsilon .
$$

The proof of the theorem will be given later in this section. As a consequence, we also have further time-regularization properties that imply uniqueness for strictly positive times as well:

Theorem 6.2. Let the assumptions of Theorem 6.1 hold (in particular, let $\delta>0$ ). Then, for any $\epsilon>0$ and any weak solution $u$,

$$
\begin{aligned}
& w \in L^{2}(\epsilon, T ; V), \\
& u \in H^{1}(\epsilon, T ; V) \cap L^{\infty}\left(\epsilon, \infty ; H^{2}(\Omega)\right) .
\end{aligned}
$$

Moreover, in the class of weak solutions, uniqueness holds at least for strictly positive times.

Remark 6.3. We point out that (6.3)- -6.4 , which suffice to prove uniqueness, are however not presumed to be optimal properties. Actually, thanks to (6.2), (1.1) is nondegenerate and (1.2) is nonsingular for strictly positive times. Thus, by means of classical methods, one could easily prove that the solution $u$ becomes, instantaneously in time, arbitrarily regular, provided of course that also the data $\gamma$ and $g$ are smooth.

As a consequence of uniqueness, we finally have

Corollary 6.4. Let the assumptions of Theorem6.1 hold (in particular, let $\delta>0$ ). Then, the dynamical process generated by weak solutions admits the (strong) global attractor $\mathcal{A}$ in the standard sense (to be more precise, in the sense of semigroups with unique continuation). Namely, $\mathcal{A}$ is a compact and fully invariant subset of $\mathcal{X}_{\mu}$ such that, for any bounded set $B \subset \mathcal{X}_{\mu}$,

$$
\lim _{t \nearrow \infty} \mathrm{d}_{\mathcal{X}}(u(t), \mathcal{A})=0,
$$

uniformly with respect to weak solutions $u$ such that $u(0) \in B$.

6.1. Proof of Theorem 6.1 in the 3D-case. We consider equation (1.2) rewritten in the form (5.19), where we assume $\delta=1$ for simplicity. We also assume $s>0$, the case $s=0$ being simpler since one can directly take advantage of the $L^{2}\left(0, T ; L^{6}(\Omega)\right)$-regularity of $w$. Then, the proof is based on a suitable version of the Moser iteration argument; i.e., we will take $\nu>1$ and test (5.19) by $\nu z^{\nu-1}$ for increasing exponents $\nu$. We have to remark that this procedure, apparently having a formal character since the above test function could grow very fast and, hence, 
have insufficient regularity, can be easily justified simply by truncating $z$ at some level $K$ and then letting $K \nearrow \infty$. In particular, the argument does not require any approximation of the equation and, hence, works for all weak solutions in the class introduced in Definition 2.3

That said, testing (5.19) by $\nu z^{\nu-1}$ and integrating over $Q_{\nu}:=\Omega \times\left(\tau_{\nu}, T\right)$, where the "initial" time $\tau_{\nu}$ will be chosen later on, we then have

$$
J_{\nu}^{\nu}+\iint_{Q_{\nu}} z^{\kappa+\nu+1} \leq\left\|z\left(\tau_{\nu}\right)\right\|_{L^{\nu}(\Omega)}^{\nu}+\nu \iint_{Q_{\nu}} \phi z^{\nu+1}-\nu \iint_{Q_{\nu}} w z^{\nu+1},
$$

where we have set

$$
J_{\nu}^{\nu}:=\|z\|_{L^{\infty}\left(\tau_{\nu}, T ; L^{\nu}(\Omega)\right)}^{\nu}+\left\|\nabla z^{\nu / 2}\right\|_{L^{2}\left(\tau_{\nu}, T ; H\right)}^{2} .
$$

Now adding

$$
\left\|z^{\nu / 2}\right\|_{L^{2}\left(\tau_{\nu}, T ; H\right)}^{2}=\|z\|_{L^{\nu}\left(Q_{\nu}\right)}^{\nu}
$$

to both sides of (6.6), in order to recover the full $V$-norm of $z^{\nu / 2}$, and setting

$$
I_{\nu}^{\nu}:=\|z\|_{L^{\infty}\left(\tau_{\nu}, T ; L^{\nu}(\Omega)\right)}^{\nu}+c_{\Omega}\|z\|_{L^{\nu}\left(\tau_{\nu}, T ; L^{3 \nu}(\Omega)\right)}^{\nu} \geq\|z\|_{L^{5 \nu / 3}\left(Q_{\nu}\right)}^{\nu},
$$

where $c_{\Omega}$ is a suitable embedding constant, we then arrive at

$$
I_{\nu}^{\nu}+\iint_{Q_{\nu}} z^{\kappa+\nu+1} \leq\left\|z\left(\tau_{\nu}\right)\right\|_{L^{\nu}(\Omega)}^{\nu}+\nu C\|z\|_{L^{\nu+1}\left(Q_{\nu}\right)}^{\nu+1}+\|z\|_{L^{\nu}\left(Q_{\nu}\right)}^{\nu}-\nu \iint_{Q_{\nu}} w z^{\nu+1},
$$

and we have to provide a bound for the last term on the right-hand side.

To do this, let $\left(p, p^{*}\right)$ and $\left(q, q^{*}\right)$ be two couples of conjugate exponents with $p \leq 2$. Then, also using (5.22), we obtain

$$
\begin{aligned}
-\nu \iint_{Q_{\nu}} w z^{\nu+1} & \leq \nu\|w\|_{L^{p}\left(\tau_{\nu}, T ; L^{q}(\Omega)\right)}\left\|z^{\nu+1}\right\|_{L^{p^{*}}\left(\tau_{\nu}, T ; L^{q^{*}}(\Omega)\right)} \\
& \leq \nu\left(\|w\|_{L^{p}\left(\tau_{\nu}, T ; L^{1}(\Omega)\right)}+\|\nabla w\|_{L^{p}\left(Q_{\nu}\right)}\right)\left\|z^{\nu+1}\right\|_{L^{p^{*}}\left(\tau_{\nu}, T ; L^{q^{*}}(\Omega)\right)} \\
& \leq \nu\left(C+\|\nabla w\|_{L^{p}\left(Q_{\nu}\right)}\right)\left\|z^{\nu+1}\right\|_{L^{p^{*}}\left(\tau_{\nu}, T ; L^{q^{*}}(\Omega)\right)},
\end{aligned}
$$

provided that we choose $q=3 p /(3-p)$, so that $W^{1, p}(\Omega) \subset L^{q}(\Omega)$ continuously.

Then, the term with $\nabla w$ is estimated this way:

$$
\begin{aligned}
\|\nabla w\|_{L^{p}\left(Q_{\nu}\right)} & =\left\|u^{s / 2} z^{s / 2} \nabla w\right\|_{L^{p}\left(Q_{\nu}\right)} \leq\left\|u^{s / 2} \nabla w\right\|_{L^{2}\left(Q_{\nu}\right)}\left\|z^{s / 2}\right\|_{L^{\frac{2 p}{2-p}}\left(Q_{\nu}\right)} \\
& \leq C\left\|z^{s / 2}\right\|_{L^{\frac{2 p}{2-p}}\left(Q_{\nu}\right)},
\end{aligned}
$$

thanks also to (5.21). Thus, collecting (6.11) and (6.12), we have

$$
-\nu \iint_{Q_{\nu}} w z^{\nu+1} \leq C \nu\left(1+\left\|z^{s / 2}\right\|_{L^{\frac{2 p}{2-p}}\left(Q_{\nu}\right)}\right)\|z\|_{L^{p^{*}(\nu+1)}\left(\tau_{\nu}, T ; L^{q^{*}(\nu+1)}(\Omega)\right)}^{\nu+1} .
$$

Now, let us assume to know a bound of the term $I_{n-1}=I_{\nu_{n-1}}$ from the preceding step of the iteration. Then, thanks to the last inequality in (6.9), we can use it to estimate the term in brackets so as to have

$$
-\nu \iint_{Q_{\nu}} w z^{\nu+1} \leq C \nu\left(1+I_{n-1}^{s / 2}\right)\|z\|_{L^{p^{*}(\nu+1)}\left(\tau_{\nu}, T ; L^{q^{*}(\nu+1)}(\Omega)\right)}^{\nu+1},
$$

provided that one chooses $p$ as follows:

$$
\frac{p}{2-p}=\frac{5 \nu_{n-1}}{3 s}, \quad \text { i.e., } \frac{1}{p}=\frac{1}{2}+\frac{3 s}{10 \nu_{n-1}} .
$$


This gives in turn

$$
\frac{1}{q}=\frac{1}{6}+\frac{3 s}{10 \nu_{n-1}}, \quad \frac{1}{p^{*}}=\frac{1}{2}-\frac{3 s}{10 \nu_{n-1}}, \quad \frac{1}{q^{*}}=\frac{5}{6}-\frac{3 s}{10 \nu_{n-1}} .
$$

Then, we have to take $\nu=\nu_{n}$ in a way suitable for the next step of the iteration. The choice is dictated by the exponents of the last term in (6.14); namely, $\nu=\nu_{n}$ should be close enough to $\nu_{n-1}$ in order that the term will still be controlled by $I_{n-1}$. Using interpolation, we require that, for some $\theta \in[0,1]$,

$$
\frac{1}{p^{*}\left(\nu_{n}+1\right)}=\frac{1-\theta}{\infty}+\frac{\theta}{\nu_{n-1}}, \quad \frac{1}{q^{*}\left(\nu_{n}+1\right)}=\frac{1-\theta}{\nu_{n-1}}+\frac{\theta}{3 \nu_{n-1}} .
$$

To compute $\theta$, we first take the quotient of the above equalities and then use (6.16). This gives

$$
\frac{3-2 \theta}{3 \theta}=\frac{p^{*}}{q^{*}}=\frac{25 \nu_{n-1}-9 s}{15 \nu_{n-1}-9 s}
$$

whence

$$
\theta=\frac{15 \nu_{n-1}-9 s}{35 \nu_{n-1}-15 s}, \quad \text { and } \quad \nu_{n}=\frac{\nu_{n-1}}{p^{*} \theta}-1=\frac{7 \nu_{n-1}-3 s-6}{6}
$$

where the second of (6.16) has also been used.

Thus, it turns out that $\nu_{n}>\nu_{n-1}$ provided that $\nu_{n-1}>3(s+2)$. Thus, in order that the above iteration could be performed, we need to find some $\bar{n} \in \mathbb{N}$ and some $\nu_{\bar{n}}>3(s+2)$ such that, for any $\epsilon \in(0,1)$,

$$
I_{\bar{n}}=\left(\|z\|_{L^{\infty}\left(\epsilon, T ; L^{\left.\nu_{\bar{n}}(\Omega)\right)}\right.}^{\nu_{\overline{\bar{n}}}}+c_{\Omega}\|z\|_{L^{\nu_{\bar{n}}\left(\epsilon, T ; L^{3 \nu \bar{n}}(\Omega)\right)}}^{\nu_{\bar{n}}}\right)^{\frac{1}{\nu_{\bar{n}}}} \leq Q\left(\epsilon^{-1}\right),
$$

where $Q$ is a computable monotone function (whose expression can depend on the magnitude of the initial data and of $T$ ).

Let us postpone the verification of (6.20) and let us now see that, for $n>\bar{n}$, the induction principle can be applied. Coming back to (6.10), we then have

$$
I_{n}^{\nu_{n}} \leq\left\|z\left(\tau_{n}\right)\right\|_{L^{\nu_{n}(\Omega)}}^{\nu_{n}}+C \nu_{n}\left(1+\|z\|_{L^{\nu_{n}+1}\left(Q_{n}\right)}^{\nu_{n}+1}\right)+C \nu_{n} I_{n-1}^{\frac{s}{2}+\nu_{n}+1},
$$

where we wrote $n$ in place of $\nu_{n}$ in some subscripts and assumed w.l.o.g. that $I_{n-1} \geq 1$. Thus, extracting the $\nu_{n}$-th root and noting that $\nu_{n}+1 \leq 5 \nu_{n-1} / 3$, we obtain

$$
I_{n} \leq\left\|z\left(\tau_{n}\right)\right\|_{L^{\nu_{n}(\Omega)}}+\left(C \nu_{n}\right)^{\frac{1}{\nu_{n}}} I_{n-1}^{\eta_{n}}, \quad \text { where } \eta_{n}:=\frac{2 \nu_{n}+s+2}{2 \nu_{n}}
$$

and $C$ is independent of $n$. Moreover, for (arbitrarily small) $\epsilon \in(0,1)$, given $\tau_{n-1}$ we can choose $\tau_{n} \in\left[\tau_{n-1}, \tau_{n-1}+\epsilon n^{-2}\right]$ such that

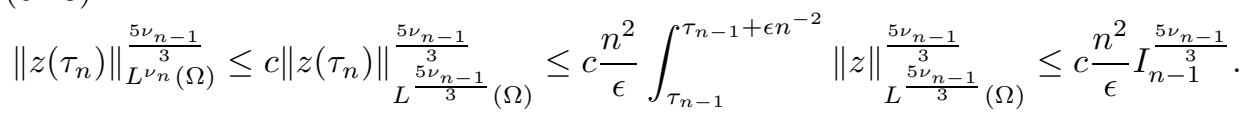

Thus, 6.22) can be rewritten as

$$
I_{n} \leq\left[\left(c \frac{n^{2}}{\epsilon}\right)^{\frac{3}{5 \nu_{n-1}}}+\left(C \nu_{n}\right)^{\frac{1}{\nu_{n}}}\right] I_{n-1}^{\eta_{n}},
$$


whence a standard computation permits us to pass to the limit w.r.t. $n \nearrow \infty$. Since $\lim _{n \nearrow_{\infty}} \tau_{n}$ exists and is less than or equal to $c \epsilon$, we then obtain

$$
\|u\|_{L^{\infty}(\Omega \times(\epsilon, T))} \leq Q\left(\epsilon^{-1}\right),
$$

for $Q$ as in (6.20), as desired.

Thus, to conclude the proof it only remains to check that (6.20) holds. To do this, we come back to (6.10) and now use the $L^{\kappa+\nu+1}$-norm to estimate the righthand side. Proceeding as above, we still arrive at (6.13), where now we have to take

$$
\frac{p}{2-p}=\frac{\kappa+\nu_{n-1}+1}{s}, \quad \text { i.e., } \frac{1}{p}=\frac{1}{2}\left(1+\frac{s}{\kappa+\nu_{n-1}+1}\right) .
$$

Thus, we obtain

$$
\begin{gathered}
\frac{1}{q}=\frac{1}{2}\left(\frac{1}{3}+\frac{s}{\kappa+\nu_{n-1}+1}\right), \quad \frac{1}{p^{*}}=\frac{1}{2}\left(1-\frac{s}{\kappa+\nu_{n-1}+1}\right), \\
\frac{1}{q^{*}}=\frac{1}{2}\left(\frac{5}{3}-\frac{s}{\kappa+\nu_{n-1}+1}\right),
\end{gathered}
$$

whence we get the analogue of (6.14), i.e.,

$$
-\nu \iint_{Q_{\nu}} w z^{\nu+1} \leq C \nu\left(1+\Lambda_{n-1}^{s / 2}\right)\|z\|_{L^{p^{*}(\nu+1)}\left(\tau_{\nu}, T ; L^{q^{*}(\nu+1)}(\Omega)\right)}^{\nu+1},
$$

where

$$
\Lambda_{\nu}^{\nu}:=\|z\|_{L^{\infty}\left(\tau_{\nu}, T ; L^{\nu}(\Omega)\right)}^{\nu}+\left\|\nabla z^{\nu / 2}\right\|_{L^{2}\left(\tau_{\nu}, T ; H\right)}^{2}+\|z\|_{L^{\nu+\kappa+1}\left(Q_{\nu}\right)}^{\nu+\kappa+1}
$$

and $\Lambda_{n}:=\Lambda_{\nu_{n}}$, as before. Then, we still have to choose $\nu=\nu_{n}$ in a suitable way. Similarly, as before, we require that for some $\theta \in[0,1]$ it is

$$
\frac{1}{p^{*}\left(\nu_{n}+1\right)}=\frac{1-\theta}{\infty}+\frac{\theta}{\kappa+\nu_{n-1}+1}, \quad \frac{1}{q^{*}\left(\nu_{n}+1\right)}=\frac{1-\theta}{\nu_{n-1}}+\frac{\theta}{\kappa+\nu_{n-1}+1} .
$$

To compute $\theta$, we first take the quotient of the above equalities and then use (6.27). This gives

$$
\frac{\theta \nu_{n-1}+(1-\theta)\left(\nu_{n-1}+\kappa+1\right)}{\theta \nu_{n-1}}=\frac{p^{*}}{q^{*}}=\frac{5\left(\nu_{n-1}+\kappa+1\right)-3 s}{3\left(\nu_{n-1}+\kappa+1-s\right)},
$$

whence

$$
\frac{1}{\theta}=1+\frac{2 \nu_{n-1}}{3\left(\nu_{n-1}+\kappa+1\right)-3 s}
$$

and, from the first of (6.30),

$$
\nu_{n}=\frac{\nu_{n-1}+\kappa+1}{\theta p^{*}}-1=\frac{5}{6} \nu_{n-1}+\frac{1}{2}(\kappa-s-1),
$$

whence it is clear that $\nu_{n}>\nu_{n-1}$ if and only if $\nu_{n-1}<3(\kappa-s-1)$. Then, proceeding similarly with the previous part of the iteration, if we start knowing a bound of $\Lambda_{\nu_{0}}$ for some $\nu_{0}>1$, then we can reach, in a finite number $\bar{n}$ of steps, any $\nu_{\bar{n}}<3(\kappa-s-1)$. Since we also need $\nu_{\bar{n}}>3(s+2)$ from before, this leads to the compatibility condition $3(s+2)<3(\kappa-s-1)$, which is equivalent to assumption (6.1). 
Thus, the proof is concluded provided that we find $\nu_{0}>1$ to start the argument. Actually, we can test (5.19) by $z^{\iota}$ for small $\iota>0$. We obtain, for $Q=\Omega \times(0, T)$,

$$
J_{1+\iota}^{1+\iota}+\iint_{Q} z^{\kappa+2+\iota} \leq\left\|z_{0}\right\|_{L^{1+\iota}(\Omega)}^{1+\iota}+\iint_{Q} \phi z^{2+\iota}-\iint_{Q} w z^{2+\iota}
$$

and, since $\kappa>3$ by (6.1), it is clear that, at least for $\iota<1$,

$$
\iint_{Q} \phi z^{2+\iota}-\iint_{Q} w z^{2+\iota} \leq \frac{1}{2} \iint_{Q} z^{\kappa+2+\iota}+C
$$

thanks also to (5.2). Hence, we can take $\nu_{0}=1+\iota$ for arbitrary $\iota \in(0,1)$, which concludes the proof in the case $d=3$.

6.2. Proof of Theorem 6.1 in the $\mathbf{2 D}$-case. The proof is carried out by the very same scheme used in the 3D case, the differences being limited to the exponents related to the use of interpolation and embeddings. Thus, we limit ourselves to point out these differences. Now, in place of (6.9), we have

$$
I_{\nu}^{\nu}:=\|z\|_{L^{\infty}\left(\tau_{\nu}, T ; L^{\nu}(\Omega)\right)}^{\nu}+\left\|z^{\frac{\nu}{2}}\right\|_{L^{2}\left(\tau_{\nu}, T ; V\right)}^{2} \geq\|z\|_{L^{2 \nu}\left(Q_{\nu}\right)}^{\nu} .
$$

Thus, taking $p \in(1,2)$, we have $q=2 p /(2-p)$, so that, to control the right-hand side of (6.14), we need to choose $p$ so that

$$
\frac{p}{2-p}=\frac{2 \nu_{n-1}}{s}, \quad \text { i.e., } \frac{1}{p}=\frac{1}{2}\left(1+\frac{s}{2 \nu_{n-1}}\right),
$$

whence we obtain

$$
\frac{1}{q}=\frac{s}{4 \nu_{n-1}}, \quad \frac{1}{p^{*}}=\frac{1}{2}\left(1-\frac{s}{2 \nu_{n-1}}\right), \quad \frac{1}{q^{*}}=1-\frac{s}{4 \nu_{n-1}}
$$

and, correspondingly,

$$
\frac{1}{p^{*}\left(\nu_{n}+1\right)}=\frac{1-\theta}{\infty}+\frac{\theta}{2 \nu_{n-1}}, \quad \frac{1}{q^{*}\left(\nu_{n}+1\right)}=\frac{1-\theta}{\nu_{n-1}}+\frac{\theta}{2 \nu_{n-1}} .
$$

Thus,

$$
\frac{2-\theta}{\theta}=\frac{p^{*}}{q^{*}}=\frac{4 \nu_{n-1}-s}{2 \nu_{n-1}-s}
$$

whence

$$
\theta=\frac{2 \nu_{n-1}-s}{3 \nu_{n-1}-s} \quad \text { and } \quad \nu_{n}=\frac{3}{2} \nu_{n-1}-\frac{s+2}{2},
$$

so that we need to find $\nu_{\bar{n}}>s+2$ in order for the procedure to work.

To do this, we proceed again as before and, choosing $p$ as in (6.26), the other exponents are then given by

$$
\frac{1}{q}=\frac{s}{2\left(\kappa+\nu_{n-1}+1\right)}, \quad \frac{1}{p^{*}}=\frac{1}{2}\left(1-\frac{s}{\kappa+\nu_{n-1}+1}\right), \quad \frac{1}{q^{*}}=1-\frac{s}{2\left(\kappa+\nu_{n-1}+1\right)} .
$$

Then, taking $\theta \in[0,1]$ as in (6.30), we now arrive at

$$
\frac{\theta \nu_{n-1}+(1-\theta)\left(\nu_{n-1}+\kappa+1\right)}{\theta \nu_{n-1}}=\frac{p^{*}}{q^{*}}=\frac{2\left(\nu_{n-1}+\kappa+1\right)-s}{\nu_{n-1}+\kappa+1-s},
$$

whence

$$
\frac{1}{\theta}=1+\frac{\nu_{n-1}}{\nu_{n-1}+\kappa+1-s} \quad \text { and } \quad \nu_{n}=\nu_{n-1}+\frac{1}{2}(\kappa-1-s),
$$


so that it is $\nu_{n}>\nu_{n-1}$ if and only if $\kappa>s+1$, i.e., (6.1) holds. Thus, we can arrive in some finite number $\bar{n}$ of steps to have $\nu_{\bar{n}}>s+2$ provided that we can start as before from $\nu_{0}=1+\iota$ for some (small) $\iota>0$. Actually, we can now take $\iota=\kappa-2$, which is strictly positive thanks to (6.1). Thus, 6.34) can be repeated without any variation and, of course, we still have (6.35) thanks to Hölder's and Young's inequalities. The proof is complete.

6.3. Proof of Theorem 6.2 and Corollary 6.4. Again, we just consider the case $d=3$, the case $d=2$ being simpler. First of all, we deduce further regularity of weak solutions. Actually, thanks to (6.2), $u$ is uniformly separated from 0 for any time $t \geq \epsilon>0, \epsilon>0$ being arbitrary. Then, (1.1) becomes in fact nondegenerate and the energy estimate gives the improved regularity (6.3). Moreover, the term $f(u)$ in (1.2) is now smooth and we can apply the linear parabolic theory (or test (1.2) by $-(t-\epsilon) \Delta u_{t}$ and perform standard computations) to deduce (6.4).

At this point, rewriting (1.1) as a family of time-dependent elliptic problems, namely

$$
-\Delta w=\frac{1}{b(u)}\left(-u_{t}+b^{\prime}(u) \nabla u \cdot \nabla w\right)
$$

relations (6.4) and (6.3) permit us to see that the right-hand side belongs to $L^{2}\left(\epsilon, T ; L^{3 / 2}(\Omega)\right)$, whence we obtain

$$
w \in L^{2}\left(\epsilon, T ; W^{2,3 / 2}(\Omega)\right) \subset L^{2}\left(\epsilon, T ; W^{1,3}(\Omega)\right) .
$$

To prove uniqueness, we can now consider a couple of solutions $u_{1}, u_{2}$, set $u:=$ $u_{1}-u_{2}$ (and, correspondingly, $w:=w_{1}-w_{2}$ ) and take the difference of equations (1.1)-(1.2) to obtain

$$
\begin{aligned}
& u_{t}-\operatorname{div}\left(b\left(u_{1}\right) \nabla w\right)=\operatorname{div}\left(\left(b\left(u_{1}\right)-b\left(u_{2}\right)\right) \nabla w_{2}\right), \\
& w=\delta u_{t}-\Delta u+W^{\prime}\left(u_{1}\right)-W^{\prime}\left(u_{2}\right),
\end{aligned}
$$

where $W^{\prime}=f+\gamma$ can be thought to be globally Lipschitz in view of the strict positivity of $u_{1}$ and $u_{2}$. Then, we test (6.47) by $w$ and (6.48) by $u_{t}$. We obtain, for some $c, \alpha>0$,

$$
\begin{aligned}
& \frac{1}{2} \frac{\mathrm{d}}{\mathrm{d} t}\|\nabla u\|^{2}+\alpha\|\nabla w\|^{2}+\delta\left\|u_{t}\right\|^{2} \\
& \quad \leq c\|u\|\left\|u_{t}\right\|+\int_{\Omega}\left|\left(b\left(u_{1}\right)-b\left(u_{2}\right)\right) \nabla w \cdot \nabla w_{2}\right|
\end{aligned}
$$

and we can estimate the last term as follows:

$$
\begin{aligned}
\int_{\Omega}\left|\left(b\left(u_{1}\right)-b\left(u_{2}\right)\right) \nabla w \cdot \nabla w_{2}\right| & \leq \frac{\alpha}{2}\|\nabla w\|^{2}+c\|u\|_{L^{6}(\Omega)}^{2}\left\|\nabla w_{2}\right\|_{L^{3}(\Omega)}^{2} \\
& \leq \frac{\alpha}{2}\|\nabla w\|^{2}+c\|u\|_{V}^{2}\left\|\nabla w_{2}\right\|_{L^{3}(\Omega)}^{2} .
\end{aligned}
$$

Thanks to (6.46), we can then apply Gronwall's Lemma to (6.49), which gives the assertion. At this point, Corollary 6.4 is an immediate consequence of the uniqueness property and of the general theory of infinite-dimensional dynamical systems [1, 34. 


\section{REFERENCES}

[1] A.V. Babin and M.I. Vishik, "Attractors of Evolution Equations". Studies in Mathematics and its Applications, 25. North-Holland Publishing Co., Amsterdam, 1992. MR.1156492 (93d:58090)

[2] J.M. Ball, Continuity properties and global attractors of generalized semiflows and the NavierStokes equations, J. Nonlinear Sci., 7 (1997), 475-502. MR.1462276 (98j:58071a)

[3] J.W. Barrett and J.F. Blowey, Finite element approximation of the Cahn-Hilliard equation with concentration dependent mobility, Math. Comp., 68 (1999), 487-517. MR.1609678 (99i:65103)

[4] J.W. Barrett, J.F. Blowey, and H. Garcke, Finite element approximation of the Cahn-Hilliard equation with degenerate mobility, SIAM J. Numer. Anal., 37 (1999), 286-318. MR.1742748 (2001c:65118)

[5] J. Becker and G. Grün, The thin-film equation: recent advances and some new perspectives, Journal of Physics: Condensed Matter, 17 (2005), S291-S307.

[6] E. Beretta, M. Bertsch and R. Dal Passo, Nonnegative solutions of a fourth-order nonlinear degenerate parabolic equation, Arch. Rational Mech. Anal., 129 (1995), 175-200. MR.1328475 (96b:35116)

[7] F. Bernis and A. Friedman, Higher order nonlinear degenerate parabolic equations, J. Differential Equations, 83 (1990), 179-206. MR.1031383 (91c:35078)

[8] A.L. Bertozzi, G. Grün, and T.P. Witelski, Dewetting films: bifurcations and concentrations, Nonlinearity, 14 (2001), 1569-1592. MR1867093 (2003m:76007)

[9] A.L. Bertozzi and M. Pugh, The lubrication approximation for thin viscous films: the moving contact line with a "porous media" cut-off of van der Waals interactions, Nonlinearity, 7 (1994), 1535-1564. MR.1304438 (95h:76036)

[10] A.L. Bertozzi and M. Pugh, Long-wave instabilities and saturation in thin film equations, Comm. Pure Appl. Math., 51 (1998), 625-661. MR.1611136 (98m:35171)

[11] F. Brezzi and G. Gilardi, FEM Mathematics, in Finite Element Handbook (H. Kardestuncer Ed.), Part I: Chapt. 1: Functional Analysis, 1.1-1.5; Chapt. 2: Functional Spaces, 2.1-2.11; Chapt. 3: Partial Differential Equations, 3.1-3.6, McGraw-Hill Book Co., New York, 1987.

[12] J.W. Cahn and J.E. Hilliard, Free energy of a nonuniform system. I. Interfacial free energy, J. Chem. Phys., 28 (1958), 258-267.

[13] V.V. Chepyzhov and M.I. Vishik, "Attractors for Equations of Mathematical Physics". American Mathematical Society Colloquium Publications 49. American Mathematical Society, Providence, RI, 2002. MR:1868930 (2003f:37001c)

[14] V.V. Chepyzhov, M.I. Vishik, and S. Zelik, A strong trajectory attractor for a dissipative reaction-diffusion system, (Russian) Dokl. Akad. Nauk 435 (2010), 155-159; translation in Dokl. Math. 82 (2010), 869-873. MR2790502 (2012c:35047)

[15] V.V. Chepyzhov, M.I. Vishik, and S. Zelik, Strong trajectory attractors for dissipative Euler equations, J. Math. Pures Appl. (9) 96 (2011), 395-407. MR2832641

[16] R. Dal Passo and H. Garcke, Solutions of a fourth order degenerate parabolic equation with weak initial trace, Ann. Scuola Norm. Sup. Pisa Cl. Sci. (4), 28 (1999), 153-181. MR 1679081 (2000b:35137)

[17] R. Dal Passo, L. Giacomelli, and A. Shishkov, The thin film equation with nonlinear diffusion, Comm. Partial Differential Equations, 26 (2001), 1509-1557. MR.1865938 (2002k:35178)

[18] A. Debussche and L. Dettori, On the Cahn-Hilliard equation with a logarithmic free energy, Nonlinear Anal., 24 (1995), 1491-1514. MR1327930 (96c:35080)

[19] A. Eden, V. Kalantarov, and S. Zelik, Infinite energy solutions for the Cahn-Hilliard equations in cylindrical domains, submitted.

[20] C.M. Elliott and H. Garcke, On the Cahn-Hilliard equation with degenerate mobility, SIAM J. Math. Anal., 27 (1996), 404-423. MR.1377481 (97c:35081)

[21] C.M. Elliott and A.M. Stuart, Viscous Cahn-Hilliard equation. II. Analysis, J. Differential Equations, 128 (1996), 387-414. MR.1398327 (97c:35080)

[22] R. Ferreira and F. Bernis, Source-type solutions to thin-film equations in higher dimensions, European J. Appl. Math., 8 (1997), 507-524. MR1479525 (98j:35150)

[23] G. Grün, Degenerate parabolic differential equations of fourth order and a plasticity model with non-local hardening, Z. Anal. Anwendungen, 14 (1995), 541-574. MR.1362530 (96m:35182) 
[24] G. Grün, On the convergence of entropy consistent schemes for lubrication type equations in multiple space dimensions, Math. Comp., 72 (2003), 1251-1279 (electronic). MR1972735 (2004c:65109)

[25] G. Grün and M. Rumpf, Simulation of singularities and instabilities arising in thin film flow, European J. Appl. Math., 12 (2001), 293-320. MR 1936040 (2003m:76050)

[26] M. Gurtin, Generalized Ginzburg-Landau and Cahn-Hilliard equations based on a microforce balance, Phys. D, 92 (1996), 178-192. MR1387065 (98m:73009)

[27] A.D. Ioffe, On lower semicontinuity of integral functionals, SIAM J. Control Optimization, 15 (1977), 521-538. MR0637234 (58:30610a)

[28] I. Moise, R. Rosa, and X. Wang, Attractors for non-compact semigroups via energy equations, Nonlinearity, 11 (1998), 1369-1393. MR.1644413 (99h:35020)

[29] A. Miranville and S. Zelik, Robust exponential attractors for Cahn-Hilliard type equations with singular potentials, Math. Methods Appl. Sci., 27 (2004), 545-582. MR2041814 (2005b:37191)

[30] A. Novick-Cohen, The Cahn-Hilliard equation: mathematical and modeling perspectives, Adv. Math. Sci. Appl., 8 (1998), 965-985. MR1657208 (99h:35190)

[31] A. Novick-Cohen and A. Shishkov, Upper bounds for coarsening for the degenerate Cahn-Hilliard equation, Discrete Contin. Dyn. Syst., 25 (2009), 251-272. MR 2525177 (2011a:35284)

[32] A. Novick-Cohen and A. Shishkov, The thin film equation with backwards second order diffusion, Interfaces Free Bound., 12 (2010), 463-496. MR.2754213

[33] G. Schimperna, Global attractors for Cahn-Hilliard equations with nonconstant mobility, Nonlinearity, 20 (2007), 2365-2387. MR2356115 (2008k:35248)

[34] R. Temam, "Infinite-Dimensional Dynamical Systems in Mechanics and Physics". SpringerVerlag, New York, 1997. MR1441312 (98b:58056)

[35] H. Wu and S. Zheng, Global attractor for the 1-D thin film equation, Asymptot. Anal., 51 (2007), 101-111. MR2311155 (2008c:35101)

Dipartimento di Matematica, Università di Pavia, Via Ferrata 1, I-27100 Pavia, Italy E-mail address: giusch04@unipv.it

Department of Mathematics, University of Surrey, Guildford, GU2 7XH, United KINGDOM

E-mail address: S.Zelik@surrey.ac.uk 